\title{
Assessment of Hybrid High-Order methods on curved meshes and comparison with discontinuous Galerkin methods ${ }^{\text {th }}$
}

\author{
Lorenzo Botti \\ Department of Engineering and Applied Sciences, University of Bergamo, Italy \\ Daniele A. Di Pietro \\ Institut Montpelliérain Alexander Grothendieck, CNRS, Univ. Montpellier, France
}

\begin{abstract}
We propose and validate a novel extension of Hybrid High-Order (HHO) methods to meshes featuring curved elements. HHO methods are based on discrete unknowns that are broken polynomials on the mesh and its skeleton. We propose here the use of physical frame polynomials over mesh elements and reference frame polynomials over mesh faces. With this choice, the degree of face unknowns must be suitably selected in order to recover on curved meshes the same convergence rates as on straight meshes. We provide an estimate of the optimal face polynomial degree depending on the element polynomial degree and on the so-called effective mapping order. The estimate is numerically validated through specifically crafted numerical tests. All test cases are conducted considering two- and three-dimensional pure diffusion problems, and include comparisons with discontinuous Galerkin discretizations. The extension to agglomerated meshes with curved boundaries is also considered.
\end{abstract}

Keywords: Hybrid High-Order methods, discontinuous Galerkin methods, curved meshes, curved elements, Poisson problem

2010 MSC: 65N08, 65N30, 65N12

\section{Introduction}

The continuous growth of high-performance computational resources and the increasing predictive capabilities of numerical models has significantly widened the range of real-life, multi-physics configurations that can be simulated. The trend is towards increasingly complex systems of partial differential equations (PDEs) in complex domains, possibly focusing on a multiscale spatial and temporal behaviour. Also the amount of physical data (permeability, mechanical properties, etc.) to be incorporated into largescale models in order to replicate the complexity encountered in the real-world is rapidly

\footnotetext{
We acknowledge the support of Agence Nationale de la Recherche through grant HHOMM (ANR15-CE40-0005) and of University of Bergamo through the project ITALY - Italian TALented Young researchers.
} 
increasing. In this context, the geometrical flexibility of numerical methods is a crucial aspect that can greatly reduce the effort required to obtain an accurate representation of both the computational domain and the problem data. We develop and numerically investigate here a specific instance of discretization methods for PDEs that support high-order approximation on curved (high-order) meshes. Curved meshes are commonly employed to provide a satisfactory representation of the domain boundary with only a moderate number of mesh elements, so that the polynomial degree can be increased while keeping the global number of degrees of freedom (DOFs) under control. The role that curved meshes play in obtaining accurate solutions when combined with high-order discretization methods has been demostrated, e.g., in [1, 2, 3].

In recent years, discretization methods supporting arbitrary approximation orders on general meshes have received an increasing amount of attention. We cite here, among others, Discontinuous Galerkin (DG) and Hybridizable Discontinuous Galerkin (HDG) methods, see e.g. 4, 5, 6, 7, Hybrid High-Order (HHO) methods 8, 9, and Virtual Element Methods (VEM) [10. The implementation of efficient DG and HDG methods on curved meshes is an open field of research. On the one hand, both Bassi et al. [5] and Warburton [11] proposed the use of polynomial spaces defined in the physical frame. In the former reference, orthonormal bases are obtained by means of a modified Gram-Schmidt procedure to ensure numerical-stability at high-polynomial degrees, while in the latter the same goal is attained by incorporating the spatial variation of the element Jacobian into the physical basis functions. On the other hand, recent works by Chan et al. [12, 13] rely on reference frame polynomial spaces introducing weightadjusted $L^{2}$-inner products in order to recover high-order accuracy. HDG has been employed on meshes with curved boundaries, mainly in the context of compressible flow problems [14, 15]; eXtended HDG with level-set description of interfaces has been recently investigated by Gurkan et al. [16]. Fidkowski [17] compared DG and HDG methods for unsteady simulations of convection-dominated flows on mapped deforming domains. Blended isogeometric DG methods formulated on elements that exactly preserve the CAD geometry have also been recently proposed in [18. Finally, we cite here the very recent work [19] of Beirão da Veiga et al. on two-dimensional Virtual Element methods supporting meshes with curved edges.

To this day, HHO methods have been essentially confined to meshes with straight edges in two space dimensions and planar faces in three space dimensions. In this work, we devise a novel extension of $\mathrm{HHO}$ methods to meshes featuring curved elements, assess its performance, and compare it with DG methods. HHO methods are based on discrete unknowns that are broken polynomials on elements and faces, and rely on two key ingredients: (i) local reconstructions obtained by solving small, embarassingly parallel problems inside each element and (ii) high-order stabilization terms penalizing face residuals. These ingredients are combined to formulate local contributions, which are then assembled as in standard finite elements. The construction is devised so that only face unknowns are globally coupled (element unknowns can be locally eliminated by static condensation), leading to global problems of relatively small size and compact stencil that can be solved efficiently, both sequentially and in parallel.

The crucial issue to extend HHO methods to curved meshes lies in the definition of face unknowns, for which we propose the use of reference frame polynomials. With this choice, the degree of face unknowns must be suitably selected in order to recover on curved meshes the same convergence rates as on straight meshes. We provide an estimate 
of the optimal face polynomial degree depending on the element polynomial degree and on the so-called effective mapping order; see 23 below. The performance of the resulting method applied to a pure diffusion problem is thoroughly assessed through a comprehensive set of tests. Specifically, the numerical results presented in Section 5 compare $h$ - and $p$-convergence rates of HHO and DG methods over two- and three-dimensional curved meshes. We consider both randomly and regularly distorted mesh sequences, which do not tend to affine meshes upon refinement, as well element subdivision mesh sequences, where mesh elements have faces that are less and less curved (asymptotically affine elements). In Section 6, we also consider $p$-convergence on curved computational domains discretized by means of agglomerated meshes in the spirit of [5].

The material is organized as follows. In Section 2 we introduce the discrete setting (mesh, mapping functions, and numerical integration). In Section 3 we discuss local polynomial spaces over elements and faces and projections thereon. The HHO and DG discretizations of the Poisson problem used for the numerical study are formulated in Section 4. The numerical results on standard curved and aggomerated meshes are collected in Sections 5 and 6 respectively. Finally, some conclusions are drawn in Section (7.

\section{Discrete setting}

In this section we discuss the main assumptions on the mesh and provide details on the functions that realize the mapping from reference geometries to physical elements, as well as on the numerical computation of integrals over elements and faces.

\subsection{Mesh}

Let $\Omega \subset \mathbb{R}^{d}, d \in\{2,3\}$, be a bounded connected open domain with Lipschitz boundary. For any $n \in\{1, \ldots, d\}$, let $\mathcal{K}^{n}$ be a fixed set of reference geometries defined in the Cartesian frame $\boldsymbol{\xi}=\left\{\xi_{i}\right\}_{1 \leqslant i \leqslant n}$. The set of reference geometries contains, e.g., triangular and quadrilateral reference elements for $n=2$, tetrahedral, hexahedral, pyramidal and prismatic reference elements for $n=3$.

We consider a possibly curved mesh $\mathcal{T}_{h}$ of $\Omega$ in the usual finite element sense, i.e., $\mathcal{T}_{h}$ is a set of disjoint open elements $T \in \mathcal{T}_{h}$ with non-empty interior that satisfy

$$
\bar{\Omega}=\sum_{T \in \mathcal{T}_{h}} \bar{T},
$$

and it holds that $h=\max _{T \in \mathcal{T}_{h}} h_{T}$ with $h_{T}$ denoting the diameter of $T$. Notice that (1) entails a simplification: more generally, $\bar{\Omega}$ is only approached as the meshsize $h$ tends to 0 , as is the case for the numerical tests of Section 6 . Mesh faces are collected in the set $\mathcal{F}_{h}$, partitioned as $\mathcal{F}_{h}=\mathcal{F}_{h}^{\mathrm{i}} \cup \mathcal{F}_{h}^{\mathrm{b}}$, where $\mathcal{F}_{h}^{\mathrm{i}}$ collects internal faces and $\mathcal{F}_{h}^{\mathrm{b}}$ boundary faces. For any mesh element $T \in \mathcal{T}_{h}$, the set $\mathcal{F}_{T}:=\left\{F \in \mathcal{F}_{h}: F \subset \partial T\right\}$ collects the mesh faces composing the boundary of $T$.

We make the following assumptions:

(i) For each $T \in \mathcal{T}_{h}$, there exists a reference element $\kappa \in \mathcal{K}^{d}$ and a polynomial mapping $\boldsymbol{\Psi}_{T}$ such that $T$ is the image of $\kappa$ through the mapping, i.e. $T=\boldsymbol{\Psi}_{T}(\kappa)$. 
(ii) For every $F \in \mathcal{F}_{h}$, there exist a reference face $\sigma \in \mathcal{K}^{d-1}$ and a polynomial mapping $\boldsymbol{\Psi}_{F}$ such that $F=\boldsymbol{\Psi}_{F}(\sigma)$.

(iii) Quadrature rules of arbitrary order are available on every reference element $\kappa \in \mathcal{K}^{n}$ and every reference face $\sigma \in \mathcal{K}^{d-1}$.

\subsection{Reference-to-physical-frame mapping functions}

For each physical element $T \in \mathcal{T}_{h}$, the reference element $\kappa \in \mathcal{K}^{d}$ is such that it has the same number of faces and nodes as $T$. The polynomial space $\mathbb{M}_{d}^{m}(\kappa), m \geqslant 1$, for each component of the mapping $\mathbf{\Psi}_{T} \in\left[\mathbb{M}_{d}^{m}(\kappa)\right]^{d}$ is such that its dimension matches the number of nodes of $\kappa$, and is chosen in the set $\left\{\mathbb{P}_{d}^{m}, \mathbb{Q}_{d}^{m}, \mathbb{S}_{d}^{m}\right\}$, where

(i) $\mathbb{P}_{d}^{m}(\kappa)$ is spanned by the restriction to $\kappa$ of the polynomial functions of $d$ variables and total degree $\leqslant m$, so that $\operatorname{dim}\left(\mathbb{P}_{d}^{m}\right)=\frac{(m+d) !}{m ! d !} ;$

(ii) $\mathbb{Q}_{d}^{m}(\kappa)$ is spanned by the restriction to $\kappa$ of polynomial functions of $d$ variables and degree $\leqslant m$ in each variable, so that $\operatorname{dim}\left(\mathbb{Q}_{d}^{m}\right)=(m+1)^{d}$;

(iii) $\mathbb{S}_{d}^{m}(\kappa)$ is the restriction to $\kappa$ of a serendipity space of polynomials of $d$ variables, i.e., any set containing all the polynomials of total degree $\leqslant m$ in each variable that can be determined uniquely by the edge and face nodes, see e.g. Brenner and Scott [20, $\S 4.6]$.

We remark that Lagrange polynomials over the set of nodes can be obtained solving linear systems involving the generalized Vandermonde matrix associated to $\mathbb{M}_{d}^{m}(\kappa)$, see e.g. Karniadakis and Sherwin [21, Section 3.3.2].

Similarly, for each physical face $F \in \mathcal{F}_{h}$, the polynomial space $\mathbb{M}_{d-1}^{m}(\sigma)$ for each component of the mapping $\boldsymbol{\Psi}_{F} \in\left[\mathbb{M}_{d-1}^{m}(\sigma)\right]^{d}$ is such that its dimension matches the number of nodes of $F$. In the case of mesh faces, $\mathbb{M}_{d-1}^{m}(\sigma)$ is chosen in the set $\left\{\mathbb{P}_{d-1}^{m}(\sigma), \mathbb{Q}_{d-1}^{m}(\sigma), \mathbb{S}_{d-1}^{m}(\sigma)\right\}$.

Throughout the rest of the paper, both the element polynomial mappings $\left\{\boldsymbol{\Psi}_{T}: T \in\right.$ $\left.\mathcal{T}_{h}\right\}$ and the face polynomial mappings $\left\{\boldsymbol{\Psi}_{F}: F \in \mathcal{F}_{h}\right\}$ are assumed to be invertible.

\subsection{Numerical integration}

Since Gaussian quadrature rules of arbitrary order are available on reference geometries, the use of reference-to-physical frame mappings is a consolidated strategy for the numerical integration of both polynomial and non-polynomial functions over mesh elements and faces.

Let $T \in \mathcal{T}_{h}$ be such that $T=\mathbf{\Psi}_{T}(\kappa)$ for some $\kappa \in \mathcal{K}^{d}$. The integral over $T$ of a function $v$ can be computed as follows:

$$
\int_{T} v(\mathbf{x}) \mathrm{d} \mathbf{x}=\int_{\kappa}\left(v \circ \boldsymbol{\Psi}_{T}\right)(\boldsymbol{\xi})\left|J_{\mathbf{\Psi}_{T}}(\boldsymbol{\xi})\right| \mathrm{d} \boldsymbol{\xi},
$$

where $\mathbf{x}$ and $\boldsymbol{\xi}$ denote, respectively, the physical and reference space coordinates, and $J_{\boldsymbol{\Psi}_{T}}$ is the Jacobian of the mapping function $\boldsymbol{\Psi}_{T}$. If $v$ is a polynomial function, the polynomial degree $q$ of the integrand in the right-hand side of (2) depends on the polynomial degrees of $v, \boldsymbol{\Psi}_{E}$ and $\left|J_{\boldsymbol{\Psi}_{T}}\right|$, say $k$ and $m$ and $j$, respectively. Since, in particular, $q=k m+j$, the degree of exactness of the quadrature rule required to compute the integral exactly 
rapidly increases when considering high polynomial orders $k$ on curved meshes (for which $m>1)$.

A similar strategy can be employed to compute the integral of a function $v$ on a face $F \in \mathcal{F}_{h}$ such that $F=\Psi_{F}(\sigma)$ for some $\sigma \in \mathcal{K}^{d-1}$ :

$$
\int_{F} v(\mathbf{x}) \mathrm{d} \mathbf{x}=\int_{\sigma}\left(v \circ \boldsymbol{\Psi}_{F}\right)(\boldsymbol{\xi})\left|J_{\Psi_{F}}(\boldsymbol{\xi})\right| \mathrm{d} \boldsymbol{\xi} .
$$

To alleviate the notation, from this point on both the integration variable and the measure are omitted from integrals.

\section{Local polynomial spaces and projections}

We discuss in this section key ingredients of both HHO and DG methods: local polynomial spaces on elements and faces and projectors thereon.

\subsection{Polynomial spaces on elements}

Physical frame polynomial spaces have been considered by several authors, e.g. Gassner et al. 4, Bassi et al. 22, and proposed as a key instrument to build DG approximations with optimal approximation properties on general polyhedral meshes, see Di Pietro and Ern [23, Chapter 1].

Let a mesh element $T \in \mathcal{T}_{h}$ and a non-negative integer $k$ be fixed. We consider the space $\mathbb{P}_{d}^{k}(T)$ spanned by the restriction to $T$ of $d$-variate polynomials of total degree $\leqslant k$. Physical frame basis functions are defined so as to inherently span the space $\mathbb{P}_{d}^{k}(T)$ even in case of arbitrarily shaped elements with possibly curved faces. From a practical point of view, in order to find a numerically satisfactory physical frame basis, we rely on the procedure described in Bassi et al. [5]: starting from a monomial basis for $\mathbb{P}_{d}^{k}(T)$ defined in a local reference frame aligned with the principal axes of inertia of $T$, an orthonormal basis is obtained by means of a modified Gram-Schmidt orthogonalization procedure.

Consider now a smooth enough real-valued function $v$. The $L^{2}$-orthogonal projection $\pi_{T}^{k} v \in \mathbb{P}_{d}^{k}(T)$ of $v$ is such that

$$
\int_{T}\left(v-\pi_{T}^{k} v\right) w=0 \text { for all } w \in \mathbb{P}_{d}^{k}(T)
$$

This relation defines uniquely the polynomial $\pi_{T}^{k} v$ as the best approximation of $v$ in $\mathbb{P}_{d}^{k}(T)$ in the $L^{2}$-norm sense.

Of course, one may choose to minimize the projection error in a different norm. A relevant choice in the present context corresponds to the elliptic projection $\varpi_{T}^{k} v \in \mathbb{P}_{d}^{k}(T)$ of $v$ that satisfies

$$
\int_{T} \nabla\left(v-\varpi_{T}^{k} v\right) \cdot \nabla w=0 \text { for all } w \in \mathbb{P}_{d}^{k}(T) \text { and } \int_{T}\left(v-\varpi_{T}^{k} v\right)=0 .
$$

These relations define a unique polynomial $\varpi_{T}^{k} v$ which is the best approximation of $v$ in $\mathbb{P}_{d}^{k}(T)$ in the sense of the $L^{2}$-norm of the gradient, and has the same mean value as $v$ over $T$. 
It has been recently proved by Di Pietro and Droniou [24, 25] that both the $L^{2}$ orthogonal and the elliptic projections approximate the function $v$ optimally inside $T$ provided that $T$ is star-shaped with respect to every point of a ball of radius comparable to $h_{T}$. This result classically extends to the case when $T$ is the finite union of starshaped subsets; see [26]. The approximation of the traces of $v$ over the faces of $T$, on the other hand, can be proved using similar arguments as in the cited references provided a standard local trace inequality is available. In what follows, we tacitly assume that the mesh sequences that we consider satisfy the requirements for both $\pi_{T}^{k} v$ and $\varpi_{T}^{k} v$ to optimally approximate both $v$ and its trace.

\subsection{Polynomial spaces on faces}

While the collection of polynomial spaces $\left\{\mathbb{P}_{d}^{k}(T): T \in \mathcal{T}_{h}\right\}$ defined above is sufficient to formulate DG methods, HHO methods also require polynomial spaces over mesh faces. Interestingly, while the faces $F \in \mathcal{F}_{h}$ are possibly $d$-dimensional entities when they belong to a curved element, the reference faces $\sigma \in \mathcal{K}^{d-1}$ are by definition $(d-1)$-dimensional entities. Accordingly, the physical frame polynomial spaces $\mathbb{P}_{d-1}^{k}(F)$ can be suitably employed only if $F \in \mathcal{F}_{h}$ is a straight line segment when $d=2$ or a subset of a plane when $d=3$ (since, in these cases, the space spanned by the restrictions to $F$ of the functions in $\mathbb{P}_{d}^{k}(T)$ is $\mathbb{P}_{d-1}^{k}(F)$ ). Polynomial spaces defined over the reference geometries appear, on the other hand, to be a natural choice for curved faces, as discussed in what follows.

For each $F \in \mathcal{F}_{h}$ such that $F=\mathbf{\Psi}_{F}(\sigma)$ for some $\sigma \in \mathcal{K}^{d-1}$ and any non-negative integer $l$, we consider the space $\mathbb{P}_{d-1}^{l}(\sigma)$ spanned by the restriction to $\sigma$ of $(d-1)$-variate polynomials. Numerically satisfactory bases for these spaces can be obtained, e.g., using Jacobi polynomials over $\sigma$. Notice that, for $d=3$, one could also consider the richer spaces $\mathbb{Q}_{d-1}^{l}(\sigma)$ (tensor product polynomials over $\sigma$ ) or $\mathbb{S}_{d-1}^{l}(\sigma)$ (serendipity polynomials over $\sigma$ ). However, we focus here on $\mathbb{P}_{d-1}^{l}(\sigma)$ since the numerical results of [27] showed that these spaces did not improve the accuracy per DOF.

A well-known phenomenon when working with reference frame polynomial spaces is that a degradation of the approximation properties with respect to physical frame polynomial spaces may be observed; see Botti [27] and the precursor work of Arnold et al. [28. Let $F \in \mathcal{F}_{h}$ denote a face such that $F=\mathbf{\Psi}_{F}(\sigma)$ for some reference face $\sigma \in \mathcal{K}^{d-1}$ with mapping $\boldsymbol{\Psi}_{F} \in\left[\mathbb{M}_{d-1}^{m}(\sigma)\right]^{d}$. The degradation of the approximation properties can be evaluated based on the so called effective mapping order $\mathrm{m}$, defined as the minimum positive integer such that

$$
\boldsymbol{\Psi}_{F} \in\left[\mathbb{P}_{d-1}^{\mathrm{m}}(\sigma)\right]^{d} .
$$

Remark 1 (Effective mapping order). The distinction between the polynomial degree $m$ of the mapping and the effective mapping order $\mathrm{m}$ reflects the (usual) implementation choice of defining the mapping space according to the number of nodes of the element; see Section 2.2. The effective mapping order corresponds the minimum degree $\mathrm{m}$ that would be required to map the reference face $\sigma$ on the physical face $F$ regarding each component of the mapping $\boldsymbol{\Psi}_{F}$ as an element of the polynomial space $\mathbb{P}_{d-1}^{m}(\sigma)$.

The effective mapping order depends on both the face shape and the choice of the polynomial space. Let us consider a few examples. Let $d=3$ and consider a six-node triangular face such that $m=2$ and $\mathbb{M}_{d-1}^{2}(\sigma)=\mathbb{P}_{d-1}^{2}(\sigma)$ (see Section 2.2 for further details). For a physical face where at least one edge is curved, the effective mapping order 
is $\mathrm{m}=2$. However, if all the edges are straight, meaning that second-order mid-edge nodes coincide with the middle point of the segments connecting vertex nodes, we have $\mathrm{m}=1$ since the second-order terms in $\boldsymbol{\Psi}_{F}$ vanish. Similarly, for a nine-node quadrangular face, corresponding to $m=2$ and $\mathbb{M}_{d-1}^{2}(\sigma)=\mathbb{Q}_{d-1}^{2}(\sigma)$, the effective mapping order can range from $m=4$ for a genuinely quadratic face to $m=1$ for a rectangular face (since three nodes are sufficient to define both triangular and rectangular elements). In conclusion, we have that

$$
\left\{\begin{array}{l}
\mathrm{m}=1 \Leftrightarrow \boldsymbol{\Psi}_{F} \text { is an affine mapping, } \\
\mathrm{m}>1 \Leftrightarrow \boldsymbol{\Psi}_{F} \text { is a nonlinear mapping, } \\
\mathrm{m}>m \Rightarrow \boldsymbol{\Psi}_{F} \text { is a nonlinear mapping and } \operatorname{dim}\left(\mathbb{M}_{d-1}^{m}\right)>\operatorname{dim}\left(\mathbb{P}_{d-1}^{m}\right) .
\end{array}\right.
$$

It is a simple matter to check that the following polynomial space inclusion holds true for any non-negative integer $k$ :

$$
\mathbb{P}_{d}^{k}(F) \circ \boldsymbol{\Psi}_{F} \subseteq \mathbb{P}_{d-1}^{k m}(\sigma),
$$

where $\mathbb{P}_{d}^{k}(F)$ is spanned by the restriction to $F$ of $d$-variate polynomials.

For a smooth enough real-valued function $v$ on $F$ and any integer $l \geqslant 0$, we define the Jacobian-weighted reference frame projection $\pi_{\sigma}^{l} v \in \mathbb{P}_{d-1}^{l}(\sigma)$ such that

$$
\int_{\sigma}\left(v \circ \mathbf{\Psi}_{F}-\pi_{\sigma}^{l} v\right) w\left|J_{\mathbf{\Psi}_{F}}\right|=0 \text { for all } w \in \mathbb{P}_{d-1}^{l}(\sigma) .
$$

This relation defines $\pi_{\sigma}^{l} v$ as the best approximation of $\left(v \circ \boldsymbol{\Psi}_{F}\right)$ in $\mathbb{P}_{d-1}^{l}(\sigma)$ in the $\left|J_{\mathbf{\Psi}_{F}}\right|^{\frac{1}{2}}$ weighted $L^{2}$-norm over $\sigma$. The approximation properties of this projection are determined by the largest integer $\mathrm{k}$ such that $\mathbb{P}_{d}^{\mathrm{k}}(F) \circ \boldsymbol{\Psi}_{F} \subseteq \mathbb{P}_{d-1}^{l}(\sigma)$. Clearly, recalling [6], we have that

$$
\mathrm{k}=\left\lfloor\frac{l}{\mathrm{~m}}\right\rfloor \text {. }
$$

Throughout the rest of this work, we assume that the following optimal trace approximation properties hold: For any $v \in H^{\mathrm{k}+1}(T)$,

$$
\left\|\pi_{\sigma}^{l} v_{\mid F} \circ \mathbf{\Psi}_{F}^{-1}-v\right\|_{L^{2}(F)} \leqslant C h_{F}^{\mathrm{k}+\frac{1}{2}}\|v\|_{H^{k+1}(T)},
$$

where $C>0$ is a real number independent of $h$ and of $F$, but possibly depending on the mesh regularity, on $l$, and on $\mathrm{m}$.

\section{Two nonconforming methods for the Poisson problem}

We formulate in this section HHO and DG discretizations of the model problem: Find $u: \Omega \rightarrow \mathbb{R}$ such that

$$
\begin{aligned}
-\triangle u=f & \text { in } \Omega, \\
u=0 & \text { on } \partial \Omega,
\end{aligned}
$$

where $f: \Omega \rightarrow \mathbb{R}$ denotes a given forcing term. This problem models diffusion phenomena where the diffusive flux is proportional to the opposite of the gradient of the scalar potential $u$. Assuming $f \in L^{2}(\Omega)$, a standard weak formulation of problem (10) reads: Find $u \in H_{0}^{1}(\Omega)$ such that

$$
\int_{\Omega} \nabla u \cdot \nabla v=\int_{\Omega} f v \text { for all } v \in H_{0}^{1}(\Omega) .
$$




\subsection{BR2 discontinuous Galerkin method}

We consider here the method of Bassi et al. 29, usually referred to as "Bassi and Rebay 2" (BR2). Let a polynomial degree $k \geqslant 1$ be fixed, and set

$$
\mathrm{U}_{h}^{k}:=\mathbb{P}_{d}^{k}\left(\mathcal{T}_{h}\right),
$$

where $\mathbb{P}_{d}^{k}\left(\mathcal{T}_{h}\right):=\left\{v \in L^{2}(\Omega): v_{\mid T} \in \mathbb{P}_{d}^{k}(T)\right.$ for all $\left.T \in \mathcal{T}_{h}\right\}$ denotes the space of broken polynomials of total degree $\leqslant k$ on the mesh $\mathcal{T}_{h}$. For all $F \in \mathcal{F}_{h}^{\mathrm{i}}$ such that $F=\partial T_{1} \cap \partial T_{2}$ (the ordering of $T_{1}, T_{2}$ is arbitrary but fixed once and for all) and all $\mathrm{v}_{h} \in \mathrm{U}_{h}^{k}$, we introduce the jump and average operators defined as follows:

$$
\llbracket \mathrm{v}_{h} \rrbracket:=\mathrm{v}_{h \mid T_{1}}-\mathrm{v}_{h \mid T_{2}}, \quad\left\{\mathrm{v}_{h}\right\}:=\frac{1}{2}\left(\mathrm{v}_{h \mid T_{1}}+\mathrm{v}_{h \mid T_{2}}\right) .
$$

On boundary faces, we conventionally set $\llbracket \mathrm{v}_{h} \rrbracket=\left\{\mathrm{v}_{h}\right\}:=\mathrm{v}_{h}$. When applied to vectorvalued functions, the jump and average operators act componentwise.

For all $F \in \mathcal{F}_{h}$, we define the local lifting operator $\mathbf{r}_{F}^{k}: L^{2}(F) \rightarrow\left[\mathbb{P}_{d}^{k}\left(\mathcal{T}_{h}\right)\right]^{d}$, such that, for all $v \in L^{2}(F)$,

$$
\int_{\Omega} \mathbf{r}_{F}^{k}(v) \cdot \boldsymbol{\tau}_{h}=\int_{F} v\left\{\boldsymbol{\tau}_{h}\right\} \cdot \mathbf{n}_{F} \text { for all } \boldsymbol{\tau}_{h} \in\left[\mathbb{P}_{d}^{k}\left(\mathcal{T}_{h}\right)\right]^{d},
$$

where $\mathbf{n}_{F}$ points out of $T_{1}$ if $F \in \mathcal{F}_{h}^{\mathrm{i}}$ is such that $F=\partial T_{1} \cap \partial T_{2}$ (the ordering of the elements is coherent with the definition of the jump), while it points out of $\Omega$ if $F \in \mathcal{F}_{h}^{\text {b }}$. We also introduce the global lifting operator $\mathbf{R}_{h}^{k}: \mathrm{U}_{h}^{k} \rightarrow\left[\mathbb{P}_{d}^{k}\left(\mathcal{T}_{h}\right)\right]^{d}$ such that, for all $\mathrm{v}_{h} \in \mathrm{U}_{h}^{k}$,

$$
\mathbf{R}_{h}^{k}\left(\mathrm{v}_{h}\right):=\sum_{F \in \mathcal{F}_{h}} \mathbf{r}_{F}^{k}\left(\llbracket \mathrm{v}_{h} \rrbracket\right) .
$$

The BR2 bilinear form on $\mathrm{U}_{h}^{k} \times \mathrm{U}_{h}^{k}$, is given by

$$
\begin{aligned}
a_{h}^{\mathrm{BR} 2}\left(\mathrm{u}_{h}, \mathrm{v}_{h}\right):= & \int_{\Omega}\left(\nabla_{h} \mathrm{u}_{h}-\mathbf{R}_{h}^{k}\left(\mathrm{u}_{h}\right)\right) \cdot\left(\nabla_{h} \mathrm{v}_{h}-\mathbf{R}_{h}^{k}\left(\mathrm{v}_{h}\right)\right) \\
& -\int_{\Omega} \mathbf{R}_{h}^{k}\left(\mathrm{u}_{h}\right) \cdot \mathbf{R}_{h}^{k}\left(\mathrm{v}_{h}\right)+\sum_{F \in \mathcal{F}_{h}} \int_{\Omega} \eta_{F} \mathbf{r}_{F}^{k}\left(\llbracket \mathrm{u}_{h} \rrbracket\right) \cdot \mathbf{r}_{F}^{k}\left(\llbracket \mathrm{v}_{h} \rrbracket\right),
\end{aligned}
$$

where $\nabla_{h}$ denotes the broken gradient on $\mathcal{T}_{h}$ and, to ensure coercivity, we take (see, e.g., [23, Lemma 5.19])

$$
\eta_{F}=1+\max _{T \in \mathcal{T}_{F}} \operatorname{card}\left(\mathcal{F}_{T}\right),
$$

where $\mathcal{T}_{F}$ collects the (one or two) elements that share $F$. The BR2 DG method for problem (10) reads: Find $\mathrm{u}_{h} \in \mathrm{U}_{h}^{k}$ such that

$$
a_{h}^{\mathrm{BR} 2}\left(\mathrm{u}_{h}, \mathrm{v}_{h}\right)=\int_{\Omega} f \mathrm{v}_{h} \text { for all } \mathrm{v}_{h} \in \mathrm{U}_{h}^{k} .
$$

It was proved by Brezzi et al. [30] on standard, straight meshes that, assuming sufficient regularity, the $L^{2}$-errors on the solution and on its gradient converge as $h^{k+1}$ and $h^{k}$, respectively; see also [23] for polyhedral meshes. A similar behaviour can be expected on the curved meshes considered here. 


\subsection{HHO method}

Let two non-negative integers $k$ and $l \geqslant k$ be given. The integer $k$ is fixed while, as it will be clear in what follows, $l$ will have to depend on both $k$ and the effective mapping order $\mathrm{m}$ (see (5)) in order to obtain optimal convergence properties. The global space of discrete unknowns for the HHO method is

$$
\underline{\mathrm{U}}_{h}^{k, l}:=\left(\underset{T \in \mathcal{T}_{h}}{X} \mathbb{P}_{d}^{k}(T)\right) \times\left(\underset{\boldsymbol{\Psi}_{F}(\sigma) \in \mathcal{F}_{h}}{\underset{\mathbb{P}_{d-1}}{X}} \mathbb{P}_{d}^{l}(\sigma)\right) .
$$

For a generic element of $\underline{\mathrm{U}}_{h}^{k, l}$, we use the classical HHO underlined notation

$$
\underline{\mathrm{v}}_{h}=\left(\left(\mathrm{v}_{T}\right)_{T \in \mathcal{T}_{h}},\left(\mathrm{v}_{\sigma}\right)_{\Psi_{F}(\sigma) \in \mathcal{F}_{h}}\right) .
$$

Given a smooth enough scalar-valued function $v$ on $\Omega$, the interpolation operator $\underline{!}_{h}^{k, l}$ returns the vector of scalar unknowns defined as follows:

$$
\underline{\mathbf{I}}_{h}^{k, l} v:=\left(\left(\pi_{T}^{k} v\right)_{T \in \mathcal{T}_{h}},\left(\pi_{\sigma}^{l} v\right)_{\boldsymbol{\Psi}_{F}(\sigma) \in \mathcal{F}_{h}}\right),
$$

where $\pi_{T}^{k}$ and $\pi_{\sigma}^{l}$ are defined by (3) and (7), respectively. The restrictions of $\underline{\mathrm{U}}_{h}^{k, l}$, $\underline{\mathrm{v}}_{h} \in \underline{\mathrm{U}}_{h}^{k, l}$, and $\underline{\underline{\mathrm{l}}}_{h}^{k, l}$ to a mesh element $T \in \mathcal{T}_{h}$ are respectively denoted by $\underline{\mathrm{U}}_{T}^{k, l}, \underline{\mathrm{v}}_{T}$, and $\underline{\mathbf{l}}_{-T}^{k, l}$.

Following Di Pietro et al. 9], for all $T \in \mathcal{T}_{h}$ we define the local potential reconstruction operator $p_{T}^{k+1}: \underline{\mathrm{U}}_{T}^{k, l} \rightarrow \mathbb{P}_{d}^{k+1}(T)$ such that, for all $\underline{\mathrm{v}}_{T}:=\left(\mathrm{v}_{T},\left(\mathrm{v}_{\sigma}\right)_{\Psi_{F}(\sigma) \in \mathcal{F}_{T}}\right) \in \underline{\mathrm{U}}_{T}^{k, l}$,

$$
\begin{array}{ll}
\int_{T} \nabla p_{T}^{k+1} \underline{\mathbf{v}}_{T} \cdot \nabla w=-\int_{T} \mathbf{v}_{T} \Delta w+\sum_{F=\Psi_{F}(\sigma) \in \mathcal{F}_{T}} \int_{F}\left(\mathbf{v}_{\sigma} \circ \Psi_{F}^{-1}\right)\left(\nabla w \cdot \mathbf{n}_{T F}\right) & \forall w \in \mathbb{P}_{d}^{k+1}(T), \\
\int_{T}\left(p_{T}^{k+1} \underline{\mathbf{v}}_{T}-\mathbf{v}_{T}\right)=0, &
\end{array}
$$

where $\mathbf{n}_{T F}$ is the unit normal to $F$ pointing out of $T$. A global potential reconstruction operator $p_{h}^{k+1}: \underline{\mathrm{U}}_{h}^{k, l} \rightarrow \mathbb{P}_{d}^{k+1}\left(\mathcal{T}_{h}\right)$ is obtained patching the elementary contributions:

$$
\left(p_{h}^{k+1} \underline{\mathbf{v}}_{h}\right)_{\mid T}:=p_{T}^{k+1} \underline{\mathbf{v}}_{T} \text { for all } T \in \mathcal{T}_{h} .
$$

Remark 2 (Approximation properties of the potential reconstruction and polynomial degree on faces). The convergence rate of the HHO method is intimately linked to the approximation properties of the potential reconstruction (see, e.g., [9, Theorem 8]), which are briefly discussed hereafter.

We start by estimating the difference between the potential reconstruction operator applied to the interpolate of a smooth function and its elliptic projection. Denote by $v$ a scalar-valued function on $T$ whose regularity will be detailed in what follows. Recalling the definitions (15) of the interpolator, (16) of the potential reconstruction, and (4) of 
the elliptic projector $\varpi_{T}^{k+1}$ we have, for all $w \in \mathbb{P}_{d}^{k+1}(T)$,

$$
\begin{aligned}
\int_{T} \nabla & \left(p_{T}^{k+1} \underline{l}_{T}^{k, l} v-\varpi_{T}^{k+1} v\right) \cdot \nabla w \\
& =-\int_{T} \pi_{T}^{k} v \Delta w+\sum_{F=\boldsymbol{\Psi}_{F}(\sigma) \in \mathcal{F}_{T}} \int_{F}\left(\pi_{\sigma}^{l} v \circ \mathbf{\Psi}_{F}^{-1}\right)\left(\nabla w \cdot \mathbf{n}_{T F}\right)-\int_{T} \nabla v \cdot \nabla w \\
& =\int_{T}\left(v-\pi_{T}^{k} v\right) \Delta w+\sum_{F=\Psi_{F}(\sigma) \in \mathcal{F}_{T}} \int_{F}\left(\pi_{\sigma}^{l} v \circ \mathbf{\Psi}_{F}^{-1}-v\right)\left(\nabla w \cdot \mathbf{n}_{T F}\right),
\end{aligned}
$$

where we have performed an integration by parts on the third term in the right-hand side to pass to the third line and we have used the definition (3) of $\pi_{T}^{k}$ together with the fact that $\triangle w \in \mathbb{P}_{d}^{k-1}(T) \subset \mathbb{P}_{d}^{k}(T)$ to cancel the first term in the right-hand side. Using the definition of the $L^{2}$-norm followed by 18 , we get

$$
\begin{aligned}
& \left\|\nabla\left(p_{T}^{k+1} \underline{-}_{T}^{k, l} v-\varpi_{T}^{k+1} v\right)\right\|_{L^{2}(T)^{d}} \\
& \quad=\sup _{w \in \mathbb{P}_{d}^{k+1}(T),\|\nabla w\|_{L^{2}(T)^{d}}=1}\left(\int_{T} \nabla\left(p_{T}^{k+1} \underline{I}_{T}^{k, l} v-\varpi_{T}^{k+1} v\right) \cdot \nabla w\right) \\
& \quad=\sup _{w \in \mathbb{P}_{d}^{k+1}(T),\|\nabla w\|_{L^{2}(T)^{d}}=1}\left(\sum_{F=\boldsymbol{\Psi}_{F}(\sigma) \in \mathcal{F}_{T}} \int_{F}\left(\pi_{\sigma}^{l} v \circ \boldsymbol{\Psi}_{F}^{-1}-v\right)\left(\nabla w \cdot \mathbf{n}_{T F}\right)\right) .
\end{aligned}
$$

Let us estimate the argument of the supremum.

(i) The case $\mathrm{m}=1$. In this case, the argument of the supremum is zero for any $l \geqslant k$. To prove it, perform a change of variables to express the integral inside the summation as an integral over the reference face $\sigma \in \mathcal{K}^{d-1}$ such that $F=\mathbf{\Psi}_{F}(\sigma)$, notice that $\left(\nabla w \cdot \mathbf{n}_{T F}\right)_{\mid F} \circ \boldsymbol{\Psi}_{F} \in \mathbb{P}_{d-1}^{k}(\sigma)$ (see (6)), and use the definition (7) of $\pi_{\sigma}^{k}$. As a consequence, we have that

$$
p_{T}^{k+1} \underline{\underline{l}}_{T}^{k, l}=\varpi_{T}^{k+1},
$$

in accordance with [9, Eq. (17)]; see also [31 (and, in particular, Section 3.1 therein) for a pedagogical introduction.

(ii) The case $\mathrm{m}>1$. For all $F \in \mathcal{F}_{T}$ such that $F=\boldsymbol{\Psi}_{F}(\sigma)$ for some $\sigma \in \mathcal{K}^{d-1}$, abridging into $a \lesssim b$ the inequality $a \leqslant C b$ with constant $C$ independent of $h$, and letting $\mathrm{k}$ be given by (8), we have

$$
\begin{aligned}
\left|\int_{F}\left(\pi_{\sigma}^{l} v \circ \mathbf{\Psi}_{F}^{-1}-v\right)\left(\nabla w \cdot \mathbf{n}_{T F}\right)\right| & \leqslant\left\|\left(\pi_{\sigma}^{l} v \circ \mathbf{\Psi}_{F}^{-1}-v\right)\right\|_{L^{2}(F)}\|\nabla w\|_{L^{2}(F)^{d}}\left\|\mathbf{n}_{T F}\right\|_{L^{\infty}(F)^{d}} \\
& \lesssim h_{T}^{\mathrm{k}+\frac{1}{2}}|v|_{H^{\mathrm{k}+1}(T)} h_{T}^{-\frac{1}{2}}\|\nabla w\|_{L^{2}(T)^{d}}
\end{aligned}
$$

where we have used the approximation properties $(9)$ of $\pi_{\sigma}^{l}$ to bound the first factor under the assumption that $v \in H^{\mathrm{k}+1}(T)$, a trace inequality for polynomials to bound the second, and the fact that the normal vector has unit Euclidian norm at every $\mathbf{x} \in F$ to estimate the third. Plugging this bound into (19), we infer that

$$
\left\|\nabla\left(p_{T}^{k+1} \underline{-}_{T}^{k, l} v-\varpi_{T}^{k+1} v\right)\right\|_{L^{2}(T)^{d}} \lesssim h_{T}^{\mathrm{k}}|v|_{H^{k+1}(T)} .
$$


In conclusion, with $a \lesssim b$ having the same meaning as above, we have that

$$
\left\|\nabla\left(p_{T}^{k+1} \underline{\underline{l}}_{T}^{k, l} v-\varpi_{T}^{k+1} v\right)\right\|_{L^{2}(T)^{d}} \lesssim \begin{cases}0 & \text { if } \mathrm{m}=1, \\ h_{T}^{\mathrm{k}}|v|_{H^{k+1}(T)} & \text { if } \mathrm{m}>1 .\end{cases}
$$

Let us now estimate the difference between $v$ and $p_{T}^{k+1} \underline{-}_{T}^{k, l} v$. Inserting $\pm \nabla \varpi_{T}^{k+1} v$ into the norm, and using the triangle inequality, we have that

$$
\left\|\nabla\left(v-p_{T}^{k+1} \underline{\mathfrak{l}}_{T}^{k, l} v\right)\right\|_{L^{2}(T)^{d}} \leqslant \underbrace{\left\|\nabla\left(v-\varpi_{T}^{k+1} v\right)\right\|_{L^{2}(T)^{d}}}_{\epsilon_{1}}+\underbrace{\left\|\nabla\left(\varpi_{T}^{k+1} v-p_{T}^{k+1} \underline{l}_{T}^{k, l} v\right)\right\|_{L^{2}(T)^{d}}}_{\epsilon_{2}} .
$$

Assuming $v \in H^{\max (k+1, \mathrm{k})+1}(T)$, and using the optimal approximation properties of the elliptic projector together with 20 , we infer the existence of two positive real numbers $C_{1}$ and $C_{2}$ independent of $h$ and of $T$ (but possibly depending on the mesh regularity, on $k, \mathrm{~m}$, and on $l$ ) such that

$$
\epsilon_{1} \leqslant C_{1} h_{T}^{k+1}|v|_{H^{k+2}(T)} \text { and } \epsilon_{2} \leqslant \begin{cases}0 & \text { if } \mathrm{m}=1 \\ C_{2} h_{T}^{\mathrm{k}}|v|_{H^{k+1}(T)} & \text { if } \mathrm{m}>1\end{cases}
$$

When $\mathrm{m}>1$, for $l$ such that $\mathrm{k}=k+1$ the two error components are equilibrated and both converge as $h_{T}^{k+1}$. For $\mathrm{m}>1$ and $l$ such that $\mathrm{k}<k+1$, on the other hand, the error component $\epsilon_{2}$ dominates. Clearly, from a computational point of view, we are interested in the smallest value of $l$ that ensures convergence in $h_{T}^{k+1}$, which corresponds to assuming that

$$
l= \begin{cases}k & \text { if } \mathrm{m}=1 \\ \mathrm{~m}(k+1) & \text { if } \mathrm{m}>1\end{cases}
$$

The HHO bilinear form on $\underline{\mathrm{U}}_{h}^{k, l} \times \underline{\mathrm{U}}_{h}^{k, l}$ is defined as follows:

$$
a_{h}^{\mathrm{HHO}}\left(\underline{\mathbf{u}}_{h}, \underline{\mathrm{v}}_{h}\right):=\int_{\Omega} \nabla_{h} p_{h}^{k+1} \underline{\mathbf{u}}_{h} \cdot \nabla_{h} p_{h}^{k+1} \underline{\mathbf{v}}_{h}+\sum_{T \in T_{h}} \sum_{F \in \mathcal{F}_{T}} \frac{1}{h_{F}} \int_{F}\left(\delta_{T F}^{l}-\delta_{T}^{k}\right) \underline{\mathbf{u}}_{T}\left(\delta_{T F}^{l}-\delta_{T}^{k}\right) \underline{\mathbf{v}}_{T},
$$

where, following [31], for all $T \in \mathcal{T}_{h}$ the difference operators $\delta_{T}^{k}$ and $\delta_{T F}^{l}, F \in \mathcal{F}_{T}$, are such that, for all $\underline{\mathrm{v}}_{T} \in \underline{\mathrm{U}}_{T}^{k, l}$,

$$
\delta_{T}^{k} \underline{\mathbf{v}}_{T}:=\pi_{T}^{k} p_{T}^{k+1} \underline{\mathbf{v}}_{T}-\mathrm{v}_{T} \text { and } \delta_{T F}^{l} \underline{\mathbf{v}}_{T}:=\left(\pi_{\sigma}^{l} p_{T}^{k+1} \underline{\mathbf{v}}_{T}-\mathbf{v}_{\sigma}\right) \circ \boldsymbol{\Psi}_{F}^{-1} \text { for all } F=\boldsymbol{\Psi}_{F}(\sigma) \in \mathcal{F}_{T} \text {. }
$$

The first term in the right-hand side of 24 is the standard Galerkin contribution responsible for consistency, whereas the second is a stabilization that ensures coercivity with respect to a suitable $H_{0}^{1}$-like discrete norm. In view of 200 , it can be easily checked that the difference operators defined by (25) vanish for $l$ as in (23) when their argument is of the form $\underline{I}_{T}^{k, l} w$ with $w \in \mathbb{P}_{d}^{k+1}(T)$. This is a crucial point to fully exploit the optimal approximation properties of the potential reconstruction in the error estimates.

The HHO method for problem $(10)$ reads: Find $\underline{u}_{h} \in \underline{\mathrm{U}}_{h}^{k, l}$ such that

$$
a_{h}^{\mathrm{HHO}}\left(\underline{\mathrm{u}}_{h}, \underline{\mathrm{v}}_{h}\right)=\sum_{T \in \mathcal{T}_{h}} \int_{T} f \mathrm{v}_{T} \text { for all } \underline{\mathrm{v}}_{h} \in \underline{\mathrm{U}}_{h}^{k, l} .
$$


It was proved in 32 on straight polygonal and polyhedral meshes that the error between $p_{h}^{k+1} \underline{\mathbf{u}}_{h}$ and the exact solution of problem 10$)$ in the $L^{2}$ - and $H_{0}^{1}$-norms converge as $h^{k+2}$ and $h^{k+1}$, respectively; see also [31, Theorem 1]. In view of [22), a similar behaviour can be expected here for $l$ as in 23.

\section{Numerical results}

In this section we numerically assess and compare the $h$ - and $p$-convergence rates of the HHO and DG discretizations of problem (10) formulated in the previous section. All the convergence plots included in this section display discretization errors on the $y$ axis versus the number of DOFs on the $x$ axis. For this reason, the axis labels are omitted. For the sake of simplicity, the convergence plots are labelled as follows:

- HHO mesh family and error type, $\mathrm{PkPl}$ : indicates the error for the HHO discretization based on the space $\underline{\mathrm{U}}_{h}^{k, l}$ (cf. (11)) over the specified mesh sequence;

- DG mesh family and error type, $P k$ : indicates the error for the DG discretization based on the space $\mathrm{U}_{h}^{k}$ (cf. (14)) over the specified mesh sequence.

The total number of DOFs is computed as follows:

DG DOFs $:=\operatorname{card}\left(\mathcal{T}_{h}\right) \times \operatorname{dim}\left(\mathbb{P}_{d}^{k}\right)=\operatorname{dim}\left(\mathrm{U}_{h}^{k}\right), \quad$ HHO DOFs $:=\operatorname{card}\left(\mathcal{F}_{h}\right) \times \operatorname{dim}\left(\mathbb{P}_{d-1}^{l}\right)$.

For the HHO discretization, the number of DOFs differs from the dimension of the space $\underline{\mathrm{U}}_{h}^{k, l}$ because the element-based DOFs can be eliminated in a preliminary step by means of static condensation. This efficient implementation strategy exploits the fact that, by construction, only face-based DOFs are globally coupled. Element-based DOFs can then be recovered from face-based DOFs in a post-processing step. A discussion on static condensation for HHO methods can be found, e.g., in [31, Section 3.2.4]; see also 33. Section 2.2.4], where more general implementation aspects are also discussed. The errors for the HHO discretization are always computed with respect to the global potential reconstruction $p_{h}^{k+1} \underline{\underline{u}}_{h}$ obtained from the discrete solution; see (17).

The global (sparse) linear systems are solved by means of iterative solvers employing right-preconditioners and imposing tight tolerances (machine precision) on the convergence of the relative residual. The local linear problems involved in the computation of the local lifting operators (12) for the DG method, as well as in the computation of the potential reconstruction (16) and in the static condensation for the HHO method, are exactly solved exactly by means of Cholesky factorizations.

\subsection{Mesh sequences}

In two space dimensions, we consider the following uniformly refined mesh sequences of the unit square $\Omega=(0,1)^{2}$ (see Figure 1 ): (i) randomly distorted six-node triangular and eight-node quadrilateral mesh families, see Figures $1 \mathrm{a}$ and $1 \mathrm{~b}$, respectively; (ii) a uniformly refined regularly distorted eight-node quadrilateral mesh family, see Figure 1c (iii) a uniformly refined element subdivision eight-node quadrilateral mesh family, see Figure 1d Uniformly refined triangular and quadrangular mesh families with straight edges are also considered for the sake of comparison. 

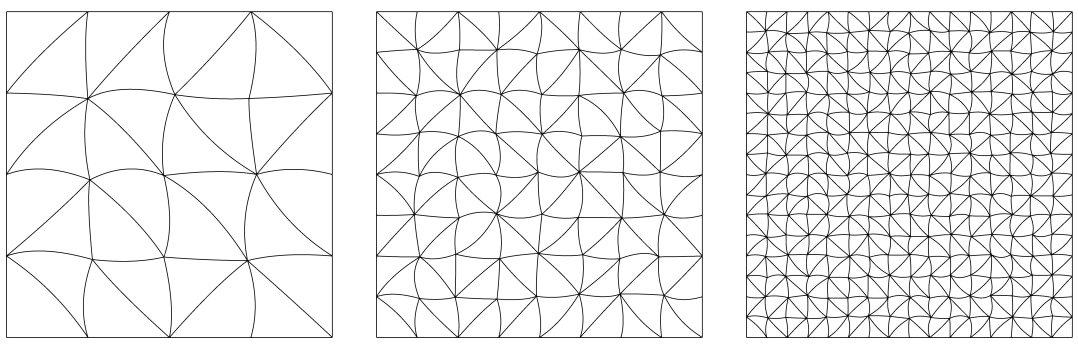

(a) Randomly distorted six-node triangular mesh sequence.
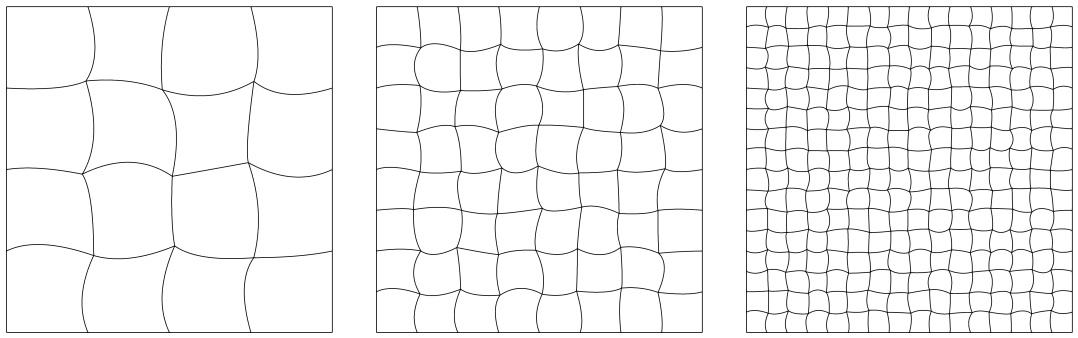

(b) Randomly distorted eight-node quadrilateral mesh sequence.
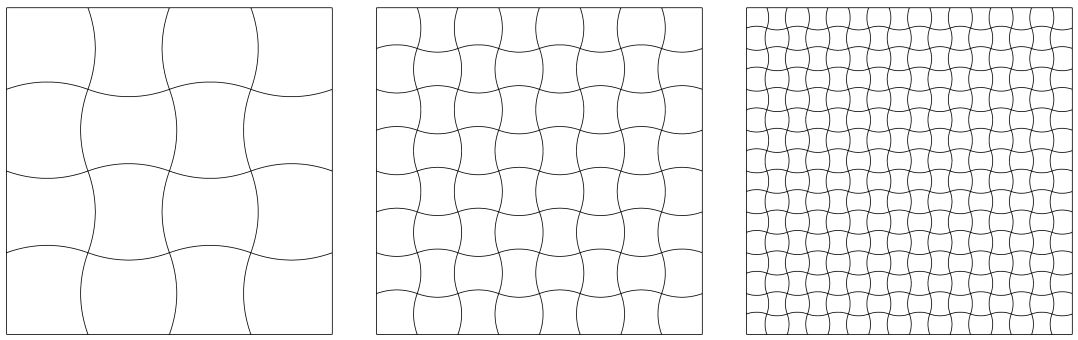

(c) Regularly distorted eight-node quadrilateral mesh sequence.
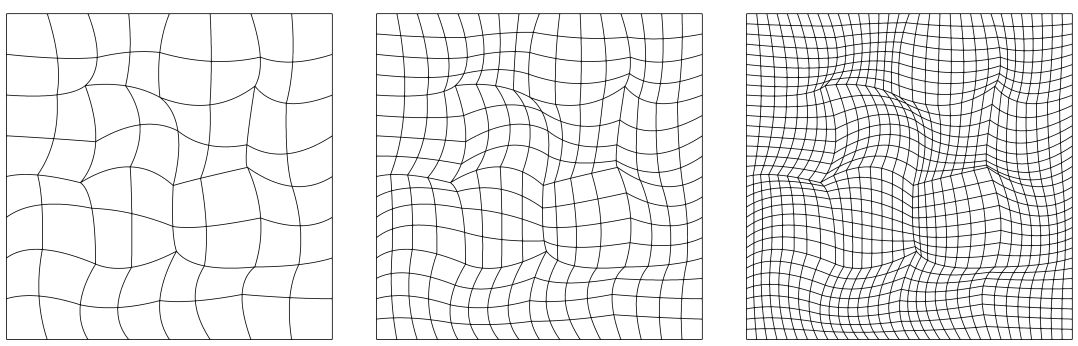

(d) Element subdivision eight-node quadrilateral mesh sequence.

Figure 1: Two-dimensional mesh sequences of $\Omega=(0,1)^{2}$. 

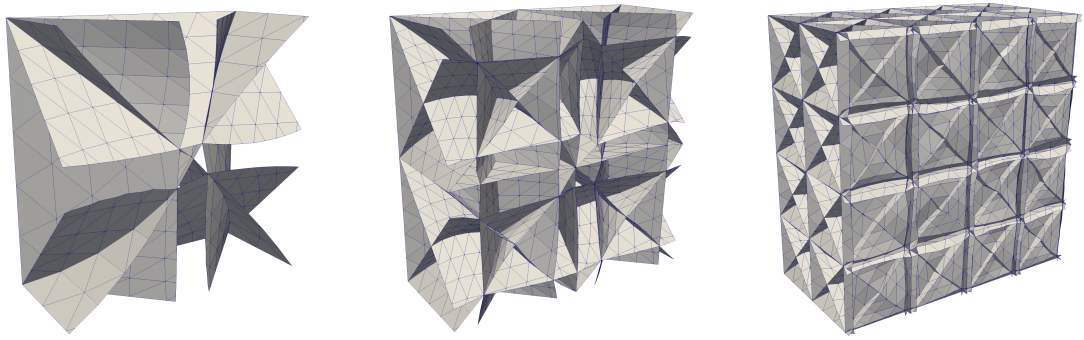

(a) Randomly distorted ten-node tetrahedral mesh sequence.
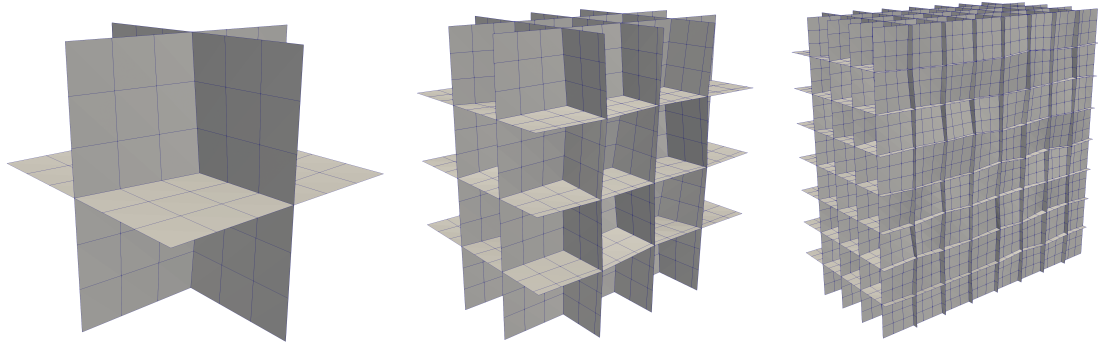

(b) Randomly distorted eight-node hexahedral mesh sequence.
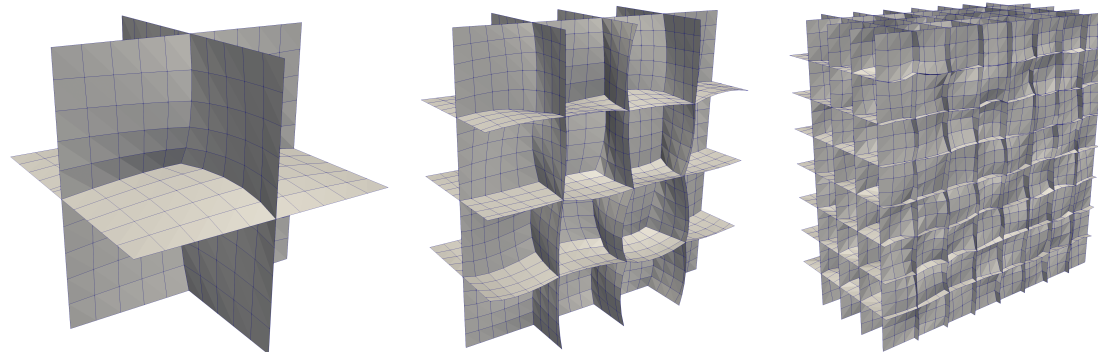

(c) Randomly distorted twenty-node hexahedral mesh sequence.

Figure 2: Three-dimensional mesh sequences of $\Omega=(0,1)^{3}$ (mesh clips showing internal faces). 


\begin{tabular}{lcccccc}
\hline Mesh sequence & Numer of nodes & $\mathbb{M}_{d}^{m}(\kappa)$ & $\mathbb{M}_{d-1}^{m}(\sigma)$ & $\mathrm{m}$ & $\lim _{h \rightarrow 0} \mathrm{~m}$ & Figure \\
\hline regular tri3 & 3 & $\mathbb{P}_{2}^{1}(\kappa)$ & $\mathbb{P}_{1}^{1}(\sigma)$ & 1 & 1 & $\overline{1}$ \\
randomDist tri6 & 6 & $\mathbb{P}_{2}^{2}(\kappa)$ & $\mathbb{P}_{1}^{2}(\sigma)$ & 2 & 2 & $1 \mathrm{a}$ \\
\hline cartesian quad4 & 4 & $\mathbb{Q}_{2}^{1}(\kappa)$ & $\mathbb{P}_{1}^{1}(\sigma)$ & 1 & 1 & $\overline{1 \mathrm{~b}}$ \\
randomDist quad8 & 8 & $\mathbb{S}_{2}^{2}(\kappa)$ & $\mathbb{P}_{1}^{2}(\sigma)$ & 2 & 2 & $\overline{1 \mathrm{c}}$ \\
regularDist quad8 & 8 & $\mathbb{S}_{2}^{2}(\kappa)$ & $\mathbb{P}_{1}^{2}(\sigma)$ & 2 & 2 & $\overline{1 \mathrm{c}}$ \\
elemSubdiv quad8 & 8 & $\mathbb{S}_{2}^{2}(\kappa)$ & $\mathbb{P}_{1}^{2}(\sigma)$ & 2 & 1 & $\overline{2 \mathrm{a}}$ \\
\hline regular tet4 & 6 & $\mathbb{P}_{3}^{1}(\kappa)$ & $\mathbb{P}_{2}^{1}(\sigma)$ & 1 & 1 & $2 \mathrm{~b}$ \\
randomDist tet10 & 10 & $\mathbb{P}_{3}^{2}(\kappa)$ & $\mathbb{P}_{2}^{2}(\sigma)$ & 2 & 2 & $2 \mathrm{c}$ \\
\hline randomDist hex8 & 8 & $\mathbb{Q}_{3}^{1}(\kappa)$ & $\mathbb{Q}_{2}^{1}(\sigma)$ & 2 & 2 & \\
randomDist hex20 & 20 & $\mathbb{S}_{3}^{2}(\kappa)$ & $\mathbb{S}_{2}^{2}(\sigma)$ & 3 & 3 & \\
\hline
\end{tabular}

Table 1: Properties of the reference-to-physical frame mappings $\mathbf{\Psi}_{T} \in\left[\mathbb{M}_{d}^{m}(\kappa)\right]^{d}$ and $\mathbf{\Psi}_{F} \in\left[\mathbb{M}_{d-1}^{m}(\sigma)\right]^{d}$ for each mesh sequence. $\mathrm{m}$ and $\lim _{h \rightarrow 0} \mathrm{~m}$ denote, respectively, the effective mapping order (see (5) ) and the asymptotic effective mapping order.

In three space dimensions, we consider uniformly refined randomly distorted tennode tetrahedral, eight-node, and twenty-node hexahedral mesh families of the unit cube $\Omega=(0,1)^{3}$ (see Figure 2). Uniformly refined tetrahedral mesh families with planar faces are also considered for the sake of comparison.

All the meshes considered here were checked to ensure positivity of the referenceto-physical frame mapping Jacobian in each mesh element. This guarantees that the quadrilaterals do not degenerate into triangular elements and that the edges do not intersect. The properties of the polynomial mappings for each mesh sequence are reported in Table 1. It is interesting to remark that $h$-refinement on the element-subdivision mesh family leads to quadrilaterals with edges that are less and less curved, which we formally indicate writing $\lim _{h \rightarrow 0} \mathrm{~m}=1\left(\lim _{h \rightarrow 0} \mathrm{~m}\right.$ should be interpreted here as an "asymptotic" effective mapping order). Notice that we do not attempt to provide a precise mathematical definition of this notion, as it seems to require some techicalities which we do not deem adequate to the present (application-oriented) paper. This topic will be further investigated in a future, theoretically-oriented work.

\subsection{HHO h-convergence rates on polynomial solutions}

In order to numerically confirm the scaling for $\epsilon_{2}$ in 22), we consider in this section manufactured polynomial solutions for which the error component $\epsilon_{1}$ vanishes (since polynomials of total degree $\leqslant(k+1)$ are invariant by $\varpi_{T}^{k+1}$-projection), and the approximation properties of $p_{T}^{k+1} \underline{-}_{T}^{k, l}$ are entirely dictated by $\epsilon_{2}$. More precisely, we define the following second and third degree polynomial solutions of the Poisson problem:

$$
u(\mathbf{x})=\sum_{i=1}^{d} x_{i}^{a}, \quad a=2,3 .
$$

The boundary conditions and forcing term are inferred from $u$. In Figures 3445 and Tables 2 3-4. we compare the results obtained on the regular (straight edge-face) and 
Table 2: Convergence rates of HHO discretizations on randomly distorted six-node triangular elements grids, see also Figure 3 Forcing term and boundary conditions imposed according to the polynomial solution 27) with $a=2$.

\begin{tabular}{lccccc}
\hline Number of elements $(32)$ & 128 & 512 & 2048 & 8192 & 32768 \\
\hline$L^{2}$ projection : $\left\|u-\pi_{h}^{k} u\right\|_{L^{2}(\Omega)}$ & 2.00 & 2.00 & 2.00 & 2.00 & 2.00 \\
\hline HHO $\mathbb{P}^{1}(T)-\mathbb{P}^{1}(\sigma):\left\|u-p_{h}^{k+1} \underline{\mathrm{u}}_{h}\right\|_{L^{2}(\Omega)}$ & 1.34 & 0.91 & 0.98 & 0.96 & 0.82 \\
HHO $\mathbb{P}^{1}(T)-\mathbb{P}^{1}(\sigma):\left\|\nabla_{h}\left(u-p_{h}^{k+1} \underline{l}_{h}^{k, l} u\right)\right\|_{L^{2}(\Omega)}{ }^{d}$ & 0.23 & -0.11 & -0.03 & 0.02 & -0.03 \\
HHO $\mathbb{P}^{1}(T)-\mathbb{P}^{1}(\sigma):\left\|\nabla_{h}\left(u-p_{h}^{k+1} \underline{\underline{u}}_{h}\right)\right\|_{L^{2}(\Omega)^{d}}$ & 0.31 & 0.05 & -0.01 & 0.02 & -0.03 \\
\hline HHO $\mathbb{P}^{1}(T)-\mathbb{P}^{2}(\sigma):\left\|u-p_{h}^{k+1} \underline{\underline{u}}_{h}\right\|_{L^{2}(\Omega)}$ & 1.88 & 1.82 & 1.87 & 2.00 & 1.98 \\
HHO $\mathbb{P}^{1}(T)-\mathbb{P}^{2}(\sigma):\left\|\nabla_{h}\left(u-p_{h}^{k+1} \underline{l}_{h}^{k, l} u\right)\right\|_{L^{2}(\Omega)^{d}}$ & 0.83 & 0.83 & 0.90 & 0.99 & 0.98 \\
HHO $\mathbb{P}^{1}(T)-\mathbb{P}^{2}(\sigma):\left\|\nabla_{h}\left(u-p_{h}^{k+1} \underline{\underline{u}}_{h}\right)\right\|_{L^{2}(\Omega)^{d}}$ & 0.87 & 0.99 & 0.87 & 0.99 & 0.98 \\
\hline HHO $\mathbb{P}^{1}(T)-\mathbb{P}^{3}(\sigma):\left\|u-p_{h}^{k+1} \underline{\mathbf{u}}_{h}\right\|_{L^{2}(\Omega)}$ & 1.41 & 1.85 & 1.91 & 1.98 & 1.98 \\
HHO $\mathbb{P}^{1}(T)-\mathbb{P}^{3}(\sigma):\left\|\nabla_{h}\left(u-p_{h}^{k+1} \underline{l}_{h}^{k, l} u\right)\right\|_{L^{2}(\Omega)^{d}}$ & 0.33 & 0.90 & 0.90 & 0.97 & 0.98 \\
HHO $\mathbb{P}^{1}(T)-\mathbb{P}^{3}(\sigma):\left\|\nabla_{h}\left(u-p_{h}^{k+1} \underline{\mathbf{u}}_{h}\right)\right\|_{L^{2}(\Omega)^{d}}$ & 0.39 & 0.92 & 0.91 & 0.95 & 0.99 \\
\hline
\end{tabular}
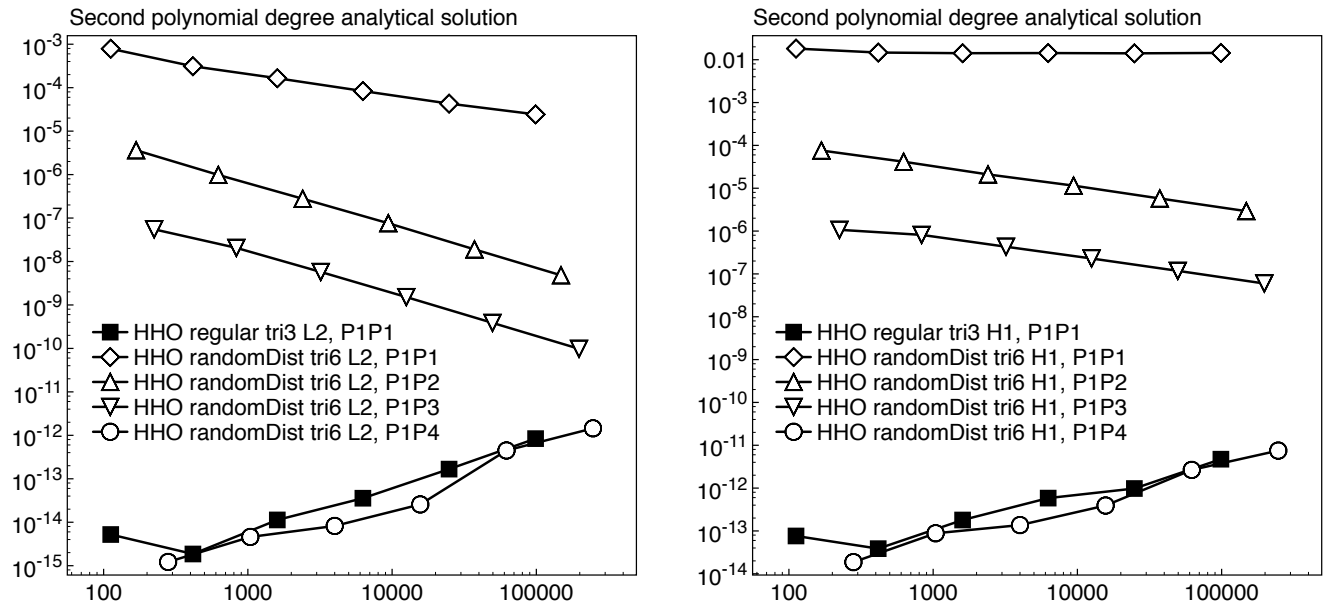

Figure 3: Error versus number of DOFs for HHO discretizations of the Poisson equation on regular threenode and randomly distorted six-node triangular elements grids. Forcing term and boundary conditions imposed according to the polynomial solution 27] with $a=2$. 
Table 3: Convergence rates of HHO discretizations on randomly distorted six-node triangular elements grids, see also Figure 4 Forcing term and boundary conditions imposed according to the polynomial solution 27 with $a=3 . *$ indicates that the convergence rate is influenced by machine precision.

\begin{tabular}{|c|c|c|c|c|c|}
\hline Number of elements (32) & 128 & 512 & 2048 & 8192 & 32768 \\
\hline$L^{2}$ projection: $\left\|u-\pi_{h}^{k} u\right\|_{L^{2}(\Omega)}$ & 2.97 & 3.00 & 3.00 & 3.00 & 3.00 \\
\hline $\operatorname{HHO} \mathbb{P}^{2}(T)-\mathbb{P}^{2}(\sigma):\left\|u-p_{h}^{k+1} \underline{\underline{u}}_{h}\right\|_{L^{2}(\Omega)}$ & 1.38 & 1.97 & 1.96 & 1.91 & 1.99 \\
\hline HHO $\mathbb{P}^{2}(T)-\mathbb{P}^{2}(\sigma):\left\|\nabla_{h}\left(u-p_{h}^{k+1} \underline{\underline{l}}_{h}^{k, l} u\right)\right\|_{L^{2}(\Omega)^{d}}$ & 0.49 & 0.86 & 1.01 & 0.91 & 1.00 \\
\hline HHO $\mathbb{P}^{2}(T)-\mathbb{P}^{2}(\sigma):\left\|\nabla_{h}\left(u-p_{h}^{k+1} \underline{\mathbf{u}}_{h}\right)\right\|_{L^{2}(\Omega)^{d}}$ & 0.42 & 0.95 & 0.96 & 0.90 & 0.99 \\
\hline $\operatorname{HHO} \mathbb{P}^{2}(T)-\mathbb{P}^{3}(\sigma):\left\|u-p_{h}^{k+1} \underline{\underline{u}}_{h}\right\|_{L^{2}(\Omega)}$ & 1.91 & 1.90 & 1.86 & 1.96 & 1.96 \\
\hline HHO $\mathbb{P}^{2}(T)-\mathbb{P}^{3}(\sigma):\left\|\nabla_{h}\left(u-p_{h}^{k+1} \underline{l}_{h}^{k, l} u\right)\right\|_{L^{2}(\Omega)^{d}}$ & 0.93 & 0.90 & 0.85 & 0.96 & 0.96 \\
\hline HHO $\mathbb{P}^{2}(T)-\mathbb{P}^{3}(\sigma):\left\|\nabla_{h}\left(u-p_{h}^{k+1} \underline{\underline{u}}_{h}\right)\right\|_{L^{2}(\Omega)^{d}}$ & 0.94 & 0.88 & 0.88 & 0.95 & 0.96 \\
\hline $\operatorname{HHO} \mathbb{P}^{2}(T)-\mathbb{P}^{4}(\sigma):\left\|u-p_{h}^{k+1} \underline{\underline{u}}_{h}\right\|_{L^{2}(\Omega)}$ & 2.36 & 2.85 & 2.85 & $2.96^{*}$ & $1.66^{*}$ \\
\hline HHO $\mathbb{P}^{2}(T)-\mathbb{P}^{4}(\sigma):\left\|\nabla_{h}\left(u-p_{h}^{k+1} \underline{\underline{l}}_{h}^{k, l} u\right)\right\|_{L^{2}(\Omega)^{d}}$ & 1.32 & 1.79 & 1.82 & 1.97 & 2.00 \\
\hline HHO $\mathbb{P}^{2}(T)-\mathbb{P}^{4}(\sigma):\left\|\nabla_{h}\left(u-p_{h}^{k+1} \underline{\mathbf{u}}_{h}\right)\right\|_{L^{2}(\Omega)^{d}}$ & 1.42 & 1.86 & 1.81 & 1.96 & 1.98 \\
\hline $\operatorname{HHO} \mathbb{P}^{2}(T)-\mathbb{P}^{5}(\sigma):\left\|u-p_{h}^{k+1} \underline{\mathbf{u}}_{h}\right\|_{L^{2}(\Omega)}$ & 1.86 & 2.75 & 2.91 & $-0.58^{*}$ & $-0.73^{*}$ \\
\hline HHO $\mathbb{P}^{2}(T)-\mathbb{P}^{5}(\sigma):\left\|\nabla_{h}\left(u-p_{h}^{k+1} \underline{l}_{h}^{k, l} u\right)\right\|_{L^{2}(\Omega)^{d}}$ & 0.87 & 1.63 & 1.96 & 1.94 & $1.99^{*}$ \\
\hline $\mathrm{HHO} \mathbb{P}^{2}(T)-\mathbb{P}^{5}(\sigma):\left\|\nabla_{h}\left(u-p_{h}^{k+1} \underline{\mathbf{u}}_{h}\right)\right\|_{L^{2}(\Omega)^{d}}$ & 0.98 & 1.63 & 2.00 & 1.90 & $1.19^{*}$ \\
\hline
\end{tabular}
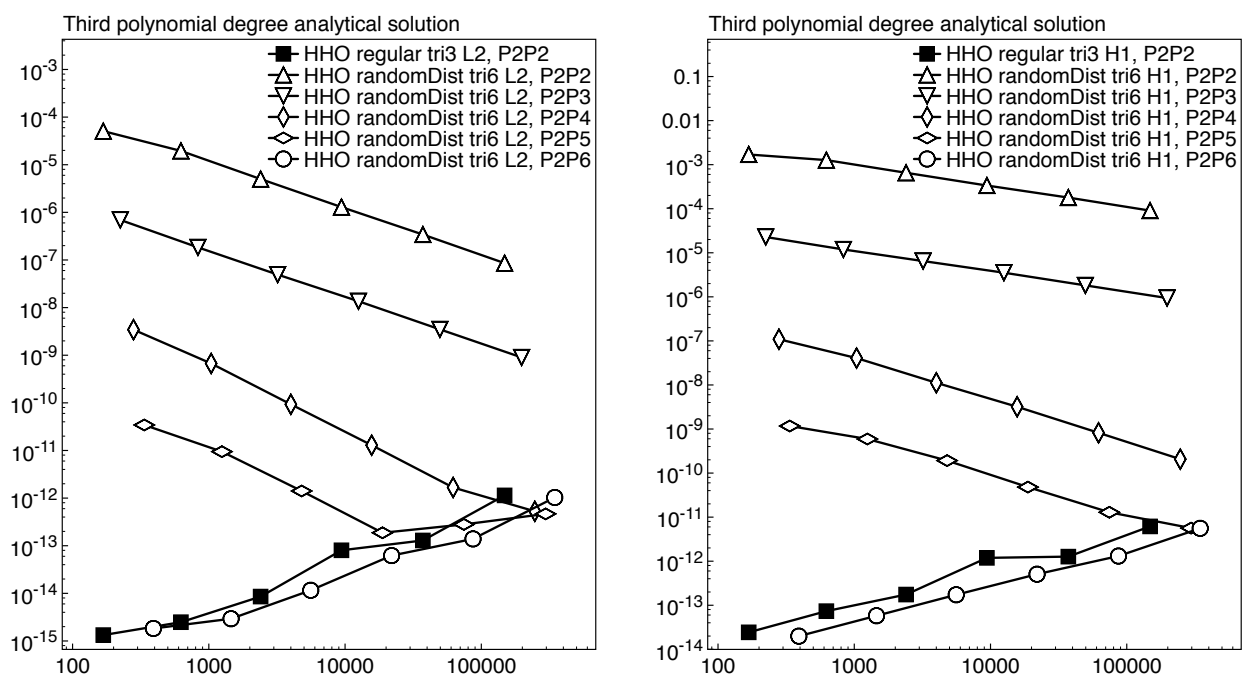

Figure 4: Error versus number of DOFs for HHO discretizations of the Poisson equation on regular threenode and randomly distorted six-node triangular elements grids. Forcing term and boundary conditions imposed according to the polynomial solution 27] with $a=3$. 
Table 4: Convergence rates of HHO discretizations on randomly distorted twenty-node hexahedral elements grids, see also Figure 5 Forcing term and boundary conditions imposed according to the polynomial solution 27) with $a=2$.

\begin{tabular}{|c|c|c|c|}
\hline Number of elements (64) & 512 & 4096 & 32768 \\
\hline$L^{2}$ projection: $\left\|u-\pi_{h}^{k} u\right\|_{L^{2}(\Omega)}$ & 1.99 & 1.99 & 1.99 \\
\hline $\operatorname{HHO} \mathbb{P}^{1}(T)-\mathbb{P}^{1}(\sigma):\left\|u-p_{h}^{k+1} \underline{\mathbf{u}}_{h}\right\|_{L^{2}(\Omega)}$ & 0.67 & 0.89 & 0.92 \\
\hline HHO $\mathbb{P}^{1}(T)-\mathbb{P}^{1}(\sigma):\left\|\nabla_{h}\left(u-p_{h}^{k+1} \underline{l}_{h}^{k, l} u\right)\right\|_{L^{2}(\Omega)^{d}}$ & -0.32 & -0.11 & -0.09 \\
\hline HHO $\mathbb{P}^{1}(T)-\mathbb{P}^{1}(\sigma):\left\|\nabla_{h}\left(u-p_{h}^{k+1} \underline{\underline{u}}_{h}\right)\right\|_{L^{2}(\Omega)^{d}}$ & -0.14 & -0.07 & -0.09 \\
\hline $\operatorname{HHO} \mathbb{P}^{1}(T)-\mathbb{P}^{2}(\sigma):\left\|u-p_{h}^{k+1} \underline{\mathrm{u}}_{h}\right\|_{L^{2}(\Omega)}$ & 0.92 & 0.88 & 0.91 \\
\hline HHO $\mathbb{P}^{1}(T)-\mathbb{P}^{2}(\sigma):\left\|\nabla_{h}\left(u-p_{h}^{k+1} \underline{L}_{h}^{k, l} u\right)\right\|_{L^{2}(\Omega)^{d}}$ & -0.11 & -0.11 & -0.11 \\
\hline HHO $\mathbb{P}^{1}(T)-\mathbb{P}^{2}(\sigma):\left\|\nabla_{h}\left(u-p_{h}^{k+1} \underline{\underline{u}}_{h}\right)\right\|_{L^{2}(\Omega)^{d}}$ & -0.06 & -0.12 & -0.08 \\
\hline $\operatorname{HHO} \mathbb{P}^{1}(T)-\mathbb{P}^{3}(\sigma):\left\|u-p_{h}^{k+1} \underline{\mathbf{u}}_{h}\right\|_{L^{2}(\Omega)}$ & 1.64 & 1.89 & 1.88 \\
\hline HHO $\mathbb{P}^{1}(T)-\mathbb{P}^{3}(\sigma):\left\|\nabla_{h}\left(u-p_{h}^{k+1} \underline{l}_{h}^{k, l} u\right)\right\|_{L^{2}(\Omega)^{d}}$ & 0.58 & 0.85 & 0.89 \\
\hline HHO $\mathbb{P}^{1}(T)-\mathbb{P}^{3}(\sigma):\left\|\nabla_{h}\left(u-p_{h}^{k+1} \underline{\mathbf{u}}_{h}\right)\right\|_{L^{2}(\Omega)^{d}}$ & 0.71 & 0.90 & 0.90 \\
\hline HHO $\mathbb{P}^{1}(T)-\mathbb{P}^{4}(\sigma):\left\|u-p_{h}^{k+1} \underline{\mathbf{u}}_{h}\right\|_{L^{2}(\Omega)}$ & 1.95 & 1.89 & 1.92 \\
\hline HHO $\mathbb{P}^{1}(T)-\mathbb{P}^{4}(\sigma):\left\|\nabla_{h}\left(u-p_{h}^{k+1} \underline{l}_{h}^{k, l} u\right)\right\|_{L^{2}(\Omega)^{d}}$ & 0.95 & 0.82 & 0.92 \\
\hline $\operatorname{HHO} \mathbb{P}^{1}(T)-\mathbb{P}^{4}(\sigma):\left\|\nabla_{h}\left(u-p_{h}^{k+1} \underline{\mathbf{u}}_{h}\right)\right\|_{L^{2}(\Omega)^{d}}$ & 1.08 & 0.89 & 0.93 \\
\hline HHO $\mathbb{P}^{1}(T)-\mathbb{P}^{5}(\sigma):\left\|u-p_{h}^{k+1} \underline{\underline{u}}_{h}\right\|_{L^{2}(\Omega)}$ & 2.14 & 1.78 & 1.87 \\
\hline HHO $\mathbb{P}^{1}(T)-\mathbb{P}^{5}(\sigma):\left\|\nabla_{h}\left(u-\bar{p}_{h}^{k+1} \underline{l}_{h}^{k, l} u\right)\right\|_{L^{2}(\Omega)^{d}}$ & 1.20 & 0.78 & 0.86 \\
\hline HHO $\mathbb{P}^{1}(T)-\mathbb{P}^{5}(\sigma):\left\|\nabla_{h}\left(u-p_{h}^{k+1} \underline{\mathbf{u}}_{h}\right)\right\|_{L^{2}(\Omega)^{d}}$ & 1.23 & 0.78 & 0.90 \\
\hline
\end{tabular}
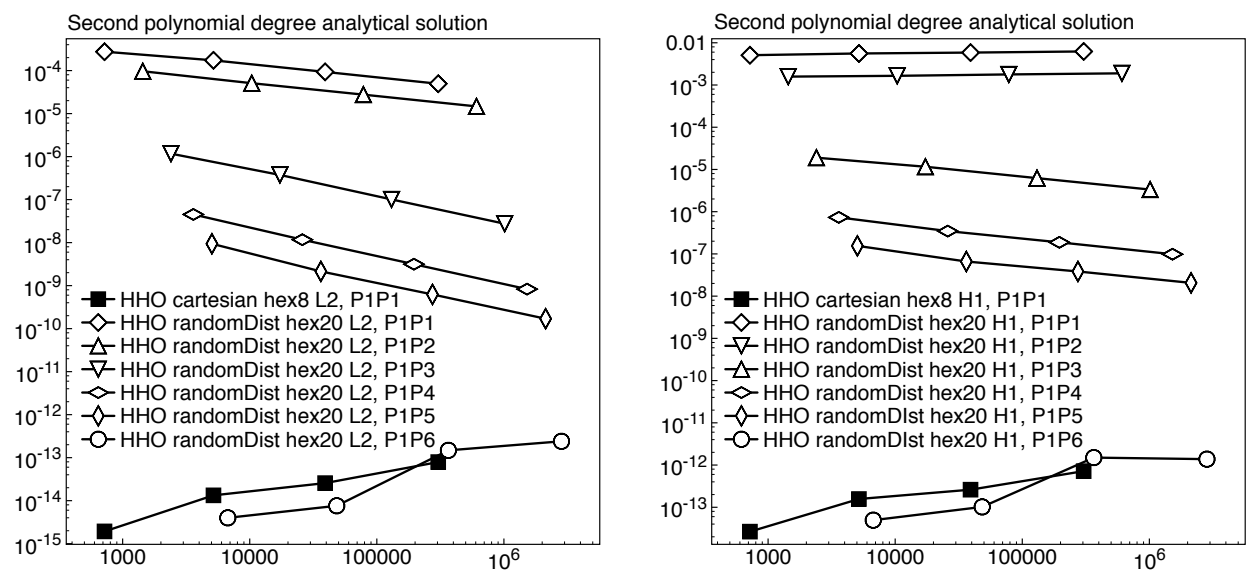

Figure 5: Error versus number of DOFs for HHO discretizations of the Poisson equation on regular eight-node and randomly distorted twenty-node hexahedral elements grids. Forcing term and boundary conditions imposed according to the polynomial solution (27) with $a=2$. 
on the randomly distorted triangular and hexahedral mesh sequences of Figures 1a 2c. Following Remark 2, the HHO method (26) should reproduce to machine precision accuracy the polynomial solutions (27) setting $k=a-1$ and $l$ as in (23). The numerical results confirm this point. More precisely:

- Regular (straight edge) triangular mesh sequence $(\mathrm{m}=1)$ : The discrete solutions obtained using the HHO spaces $\underline{\mathrm{U}}_{h}^{1,1}$ and $\underline{\mathrm{U}}_{h}^{2,2}$ are exact up to machine precision for $a=2$ and $a=3$, respectively;

- Randomly distorted triangular mesh sequence $(\mathrm{m}=2)$ : The discrete solutions obtained using the HHO spaces $\underline{\mathrm{U}}_{h}^{1,4}$ and $\underline{\mathrm{U}}_{h}^{2,6}$ are exact up to machine precision for $a=2$ and $a=3$, respectively.

- Regular (straight face) hexahedral mesh sequence $(\mathrm{m}=1)$ : The discrete solutions obtained using the HHO spaces $\underline{\mathrm{U}}_{h}^{1,1}$ are exact up to machine precision for $a=2$;

- Randomly distorted hexahedral mesh sequence $(\mathrm{m}=3)$ : The discrete solutions obtained using the HHO spaces $\underline{\cup}_{h}^{1,6}$ are exact up to machine precision for $a=2$.

Moreover, if $l$ is such that $\mathrm{k}<k+1$, we observe the expected rates of $h^{\mathrm{k}+1}$ for the $L^{2}$ error on the solution and $h^{\mathrm{k}}$ for the $L^{2}$-error on the gradient; see Tables 24 . Note that, since $\mathrm{m}=2$ for the randomly distorted triangular mesh sequence, the choices $l=2,3$ and $l=4,5$ yield the same convergence rates. Similarly, since $\mathrm{m}=3$ for the randomly distorted hexahedral mesh sequence, the choices $l=1,2$ and $l=3,4,5$ yield the same convergence rates.

We can also remark from Tables 24 (where $\pi_{h}^{k}$ denotes the global $L^{2}$-orthogonal projector on $\mathrm{U}_{h}^{k}$ ) that the reconstruction of the interpolated exact solution behaves as the reconstruction of the discrete solution in terms of gradient errors: compare, e.g., $\left\|\nabla_{h}\left(u-p_{h}^{k+1} \underline{\underline{l}}_{h}^{k, l} u\right)\right\|_{L^{2}(\Omega)^{d}}$ with $\left\|\nabla_{h}\left(u-p_{h}^{k+1} \underline{\mathbf{u}}_{h}\right)\right\|_{L^{2}(\Omega)^{d}}$. In other words, the discretization error for the HHO discretization scales optimally with respect to the approximation properties of $p_{h}^{k+1} \underline{l}_{h}^{k, l}$.

\section{3. h-convergence rates on trigonometric solutions}

In order to investigate and compare the $h$-convergence rates of HHO and DG methods, we focus on a trigonometric solution of the Poisson equation. This choice is more representative of the performance of the methods in real-life computations. In particular, unlike the previous section, both error components in the right-hand side of (21) are non-zero, so that the relative magnitude of the constants $C_{1}$ and $C_{2}$ in $(22)$ determines the observed convergence rate for $\mathrm{HHO}$ discretizations before the asymptotic regime is reached. We compute the forcing term and boundary conditions of the Poisson problem according to the following analytical solution:

$$
u(\mathbf{x})=\prod_{i=1}^{d} \sin \left(\pi x_{i}\right) .
$$

We first point out that DG discretizations based on the space $\mathrm{U}_{h}^{k}$ provide convergence rates of $h^{k+1}$ for the $L^{2}$-error on the solution and $h^{k}$ for the $L^{2}$-error on its gradient, both on regular and distorted curved mesh sequences. Therefore, in all the plots hereafter, 

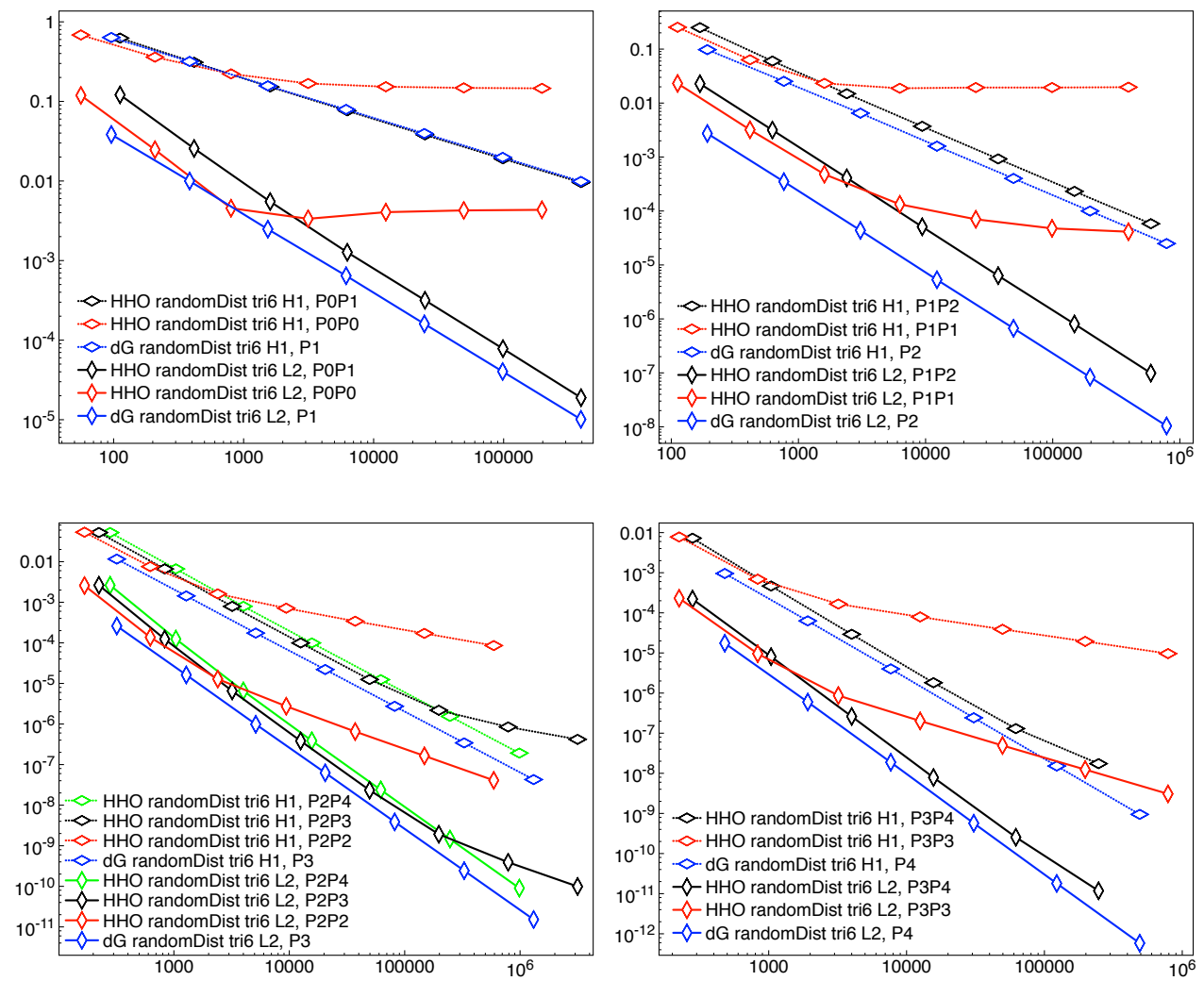

Figure 6: Error versus number of DOFs for HHO and DG discretizations of the Poisson equation over uniformly refined triangular mesh sequences. Forcing term and boundary conditions imposed according to the trigonometric solution 28 . 
the convergence rates of DG solutions are considered as a reference. HHO discretizations on triangular mesh families perform significantly better in terms of $h$-convergence rates when approximating a trigonometric potential instead of a polynomial one, see Figure 6 and compare with the results of Section 5.2. In particular, HHO formulations based on the space $\underline{\mathrm{U}}_{h}^{k, k+1}$ yield optimal convergence rates on the randomly distorted mesh family in all the cases but $k=2$, where $l=k+2$ is required. This behavior suggests that $C_{1} \gg C_{2}$ in (22) and, accordingly, the error component $\epsilon_{1}$ in (21) dominates during the early stages of the convergence history, while $\epsilon_{2}$ shows up at latter stages due to its weaker rate of convergence; see $(22)$.

Similarly, the HHO spaces $\underline{\bigcup}_{h}^{k, k+1}$ yield optimal convergence rates on randomly distorted quadrilateral mesh families, see Figures 7,8 and 9,10 . Note that the randomly distorted mesh sequence is associated with an earlier and more pronounced convergence degradation with respect to the regularly distorted mesh family, even if the asymptotic convergence rate is the same. Interestingly, no convergence degradation is observed for equal-order HHO spaces $\underline{\mathrm{U}}_{h}^{k, k}$ on the element subdivision mesh family. This behaviour has been documented by Botti 27] and Bassi et al. 34] in the context of reference frame DG discretizations, and can be explained taking into account the asymptotic effective mapping order. Indeed, whenever mesh refinement drives towards mesh elements with edges that are less and less curved (i.e., when $\lim _{h \rightarrow 0} \mathrm{~m}=1$ ), optimal convergence rates can be expected for the equal-order spaces $\underline{U}_{h}^{k, k}$; see [27, Remark 1] on this subject.

Convergence degradation is also observed in three space dimensions when considering the equal-order HHO spaces $\underline{\mathrm{U}}_{h}^{k, k}$ over the randomly distorted ten-node tetrahedral mesh family and the randomly distorted twenty-node hexahedral mesh sequence. Once again, increasing the polynomial degree by one over mesh faces (i.e., taking $l=k+1$ ) is usually sufficient to recover optimal $h$-convergence rates, see Figures $11 \mid 12$ and Figures 13 .14. Note that one could have expected a more significant degradation of the convergence properties than observed on the distorted twenty-node hexahedral elements (for which we have $\mathrm{m}=3$ ).

The numerical results presented in this section demonstrate that DG discretizations based on the space $\mathrm{U}_{h}^{k+1}$ and HHO discretization based on the space $\underline{\mathrm{U}}_{h}^{k, k}$ provide similar convergence rates on good mesh sequence with asymptotic effective mapping order equal to 1 . This is crucial considering that good mesh generators should drive towards affine polynomial mappings upon mesh refinement, even in case of complex domain boundaries. In this context, the possibility to consider $k=0$ in HHO formulations on arbitrarily shaped mesh families can be considered a significant advantage with respect to DG discretizations whenever low-order methods are mandatory to keep the computational cost low. We notice, in passing, that the HHO formulation with $k=0$ enters the HybridMixed-Mimetic framework of [35; 9, Section 2.5] for further details. In the context of Mimetic Finite Differences, a treatment of curved faces has been proposed in 36, where similar results are found for mesh faces that become less and less curved. As opposite, in the presence of randomly distorted mesh elements (that might arise, e.g., in Lagrangian and ALE computations), DG seems to be a safer choice.

\section{4. p-convergence on trigonometric solutions}

When sufficient regularity of the exact solution can be expected, the possibility to improve the solution accuracy by increasing the polynomial degree ( $p$-refinement) is a 
-.. HHO cartesian quad4 $\mathrm{H} 1, \mathrm{POPO}$ $-\mathrm{O}-\mathrm{HHO}$ elemSubdiv quad8 $\mathrm{H} 1, \mathrm{POPO}$ $\cdots \cdots \cdot \mathrm{HHO}$ randomDist quad8, $\mathrm{H} 1, \mathrm{POPO}$ $\ldots . . . \mathrm{HHO}$ regularDist quad8 $\mathrm{H} 1, \mathrm{P0P0}$

dG cartesian quad4 $\mathrm{H} 1, \mathrm{P} 1$

-.. dG elemSubdiv quad8 $\mathrm{H} 1, \mathrm{P} 1$ $\cdots \cdots \mathrm{dG}$ randomDist quad8 $\mathrm{H} 1, \mathrm{P}$ ... $\cdots \mathrm{dG}$ regularDist quad8 $\mathrm{H} 1, \mathrm{P} 1$ - $\mathrm{HHO}$ cartesian quad4 L2, POPO $-\mathrm{O}-\mathrm{HHO}$ elemSubdiv quad8 $\mathrm{L} 2, \mathrm{POPO}$ $-\nabla-\mathrm{HHO}$ randomDist quad8 $\mathrm{L} 2, \mathrm{POPO}$ $\checkmark-\mathrm{HHO}$ regularDist quad8 $\mathrm{L} 2, \mathrm{POPO}$

- dG cartesian quad4 L2, P1 $-\mathrm{O}-\mathrm{dG}$ elemSubdiv quad8 $\mathrm{L} 2, \mathrm{P} 1$ $-\nabla-\mathrm{dG}$ randomDist quad8 $L 2, \mathrm{P} 1$ $\checkmark-d G$ regularDist quad8 $L 2, P 1$

- $\mathrm{HHO}$ cartesian quad4 H1, P1P1 $\mathrm{O}-\mathrm{HHO}$ elemSubdiv quad8 $\mathrm{H} 1, \mathrm{P} 1 \mathrm{P} 1$ $--\nabla-\mathrm{HHO}$ randomDist quad8 $\mathrm{H} 1, \mathrm{P} 1 \mathrm{P} 1$ $--\checkmark-\mathrm{HHO}$ regularDist quad8 $\mathrm{H} 1, \mathrm{P} 1 \mathrm{P} 1$ - dG cartesian quad4 H1, P2 -O-dG elemSubdiv quad8 $\mathrm{H} 1, \mathrm{P} 2$ $-\nabla-\mathrm{dG}$ randomDist quad8 $\mathrm{H1}, \mathrm{P} 2$ $--\checkmark-\mathrm{dG}$ regularDist quad8 $\mathrm{H1}, \mathrm{P} 2$ - HHO cartesian quad4 L2, P1P1 $-\mathrm{O}-\mathrm{HHO}$ elemSubdiv quad8 L2, P1P1 $-\nabla-\mathrm{HHO}$ randomDist quad8 $\mathrm{L} 2, \mathrm{P} 1 \mathrm{P} 1$ $\checkmark-\mathrm{HHO}$ regularDist quad8 L2, P1P1 $-\mathrm{dG}$ cartesian quad4 L2, P2

$-O-d G$ elemSubdiv quad8 $L 2, P 2$ $-\nabla-\mathrm{dG}$ randomDist qaud8 $\mathrm{L} 2, \mathrm{P} 2$

$\checkmark \mathrm{dG}$ regularDist quad8 $\mathrm{L} 2, \mathrm{P} 2$
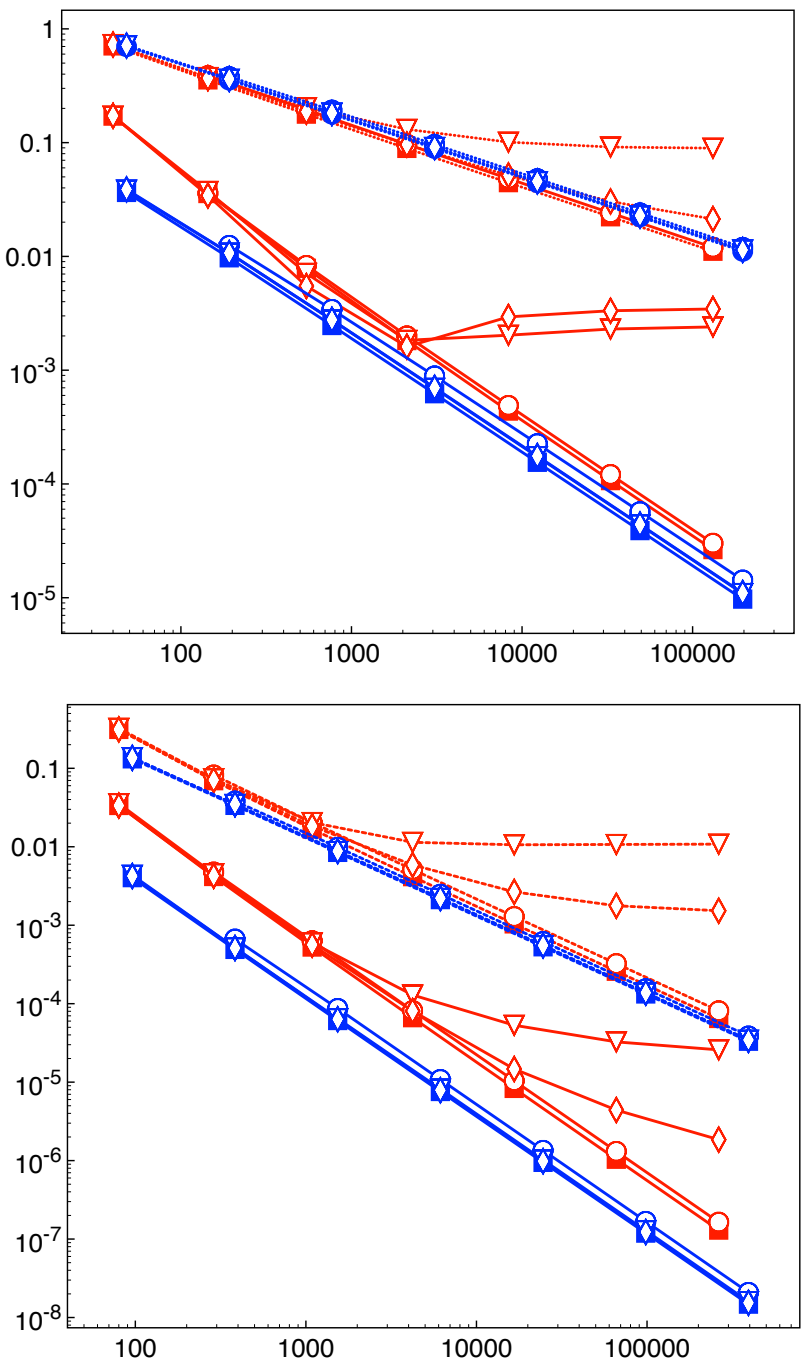

Figure 7: Error versus number of DOFs for HHO and DG discretizations of the Poisson equation over uniformly refined quadrilateral meshes. Forcing term and boundary conditions imposed according to the trigonometric solution $[28$. 
- $\mathrm{HHO}$ cartesian quad4 $\mathrm{H} 1, \mathrm{P} 2 \mathrm{P} 2$ -O- $\mathrm{HHO}$ elemSubdiv quad8 $\mathrm{H} 1, \mathrm{P} 2 \mathrm{P} 2$ $--\nabla-\mathrm{HHO}$ randomDist quad8 $\mathrm{H1}, \mathrm{P} 2 \mathrm{P} 2$ $---\mathrm{HHO}$ regularDist quad8 $\mathrm{H} 1, \mathrm{P} 2 \mathrm{P} 2$

- dG cartesian quad4 $\mathrm{H} 1, \mathrm{P} 3$ -O-dG elemSubdiv quad8 $\mathrm{H} 1, \mathrm{P} 3$ $-\nabla-\mathrm{dG}$ randomDist quad8 $\mathrm{H1}, \mathrm{P3}$ -- $\downarrow-\mathrm{dG}$ regularDist quad8 $\mathrm{H} 1, \mathrm{P} 3$ - $\mathrm{HHO}$ cartesian quad4 L2, P2P2 $-\mathrm{O}-\mathrm{HHO}$ elemSubdiv quad8 L2, P2P2 $-\nabla-\mathrm{HHO}$ randomDist quad8 $\mathrm{L} 2, \mathrm{P} 2 \mathrm{P} 2$ $\checkmark-\mathrm{HHO}$ regularDist quad8 $\mathrm{L} 2, \mathrm{P} 2 \mathrm{P} 2$

dG cartesian quad4 L2, P3 $-d G$ elemSubdiv quad8 $L 2, P 3$ $-\nabla-d G$ randomDist quad8 $L 2, P 3$ $\checkmark$ dG regularDist quad8 $L 2, P 3$

- $\mathrm{HHO}$ cartesian quad4 $\mathrm{H} 1, \mathrm{P} 3 \mathrm{P} 3$ $\mathrm{O}-\mathrm{HHO}$ elemSubdiv quad8 $\mathrm{H} 1, \mathrm{P} 3 \mathrm{P} 3$ $--\nabla-\mathrm{HHO}$ randomDist quad8 $\mathrm{H} 1, \mathrm{P} 3 \mathrm{P} 3$ $-\checkmark-\mathrm{HHO}$ regularDist quad8 $\mathrm{H1}, \mathrm{P3P} 3$ - dG cartesian quad4 $\mathrm{H} 1, \mathrm{P} 4$ -O- dG elemSubdiv quad8 $\mathrm{H} 1, \mathrm{P} 4$ $--\nabla-\mathrm{dG}$ randomDist quad8 $\mathrm{H} 1, \mathrm{P} 4$ $-\checkmark-\mathrm{dG}$ regularDist quad8 $\mathrm{H1}, \mathrm{P} 4$ - HHO cartesian quad4 L2, P3P3 $-\mathrm{O}-\mathrm{HHO}$ elemSubdiv quad8 L2, P3P3 $-\nabla-\mathrm{HHO}$ randomDist quad8 $\mathrm{L} 2, \mathrm{P} 3 \mathrm{P} 3$ $\checkmark-\mathrm{HHO}$ regularDist quad8 L2, P3P3 - dG cartesian quad4 L2, P4 -O- dG elemSubdiv quad8 L2, P4 $-\nabla-\mathrm{dG}$ randomDist quad8 L2, P4 $\checkmark \mathrm{dG}$ regularDist quad8 $\mathrm{L} 2, \mathrm{P} 4$
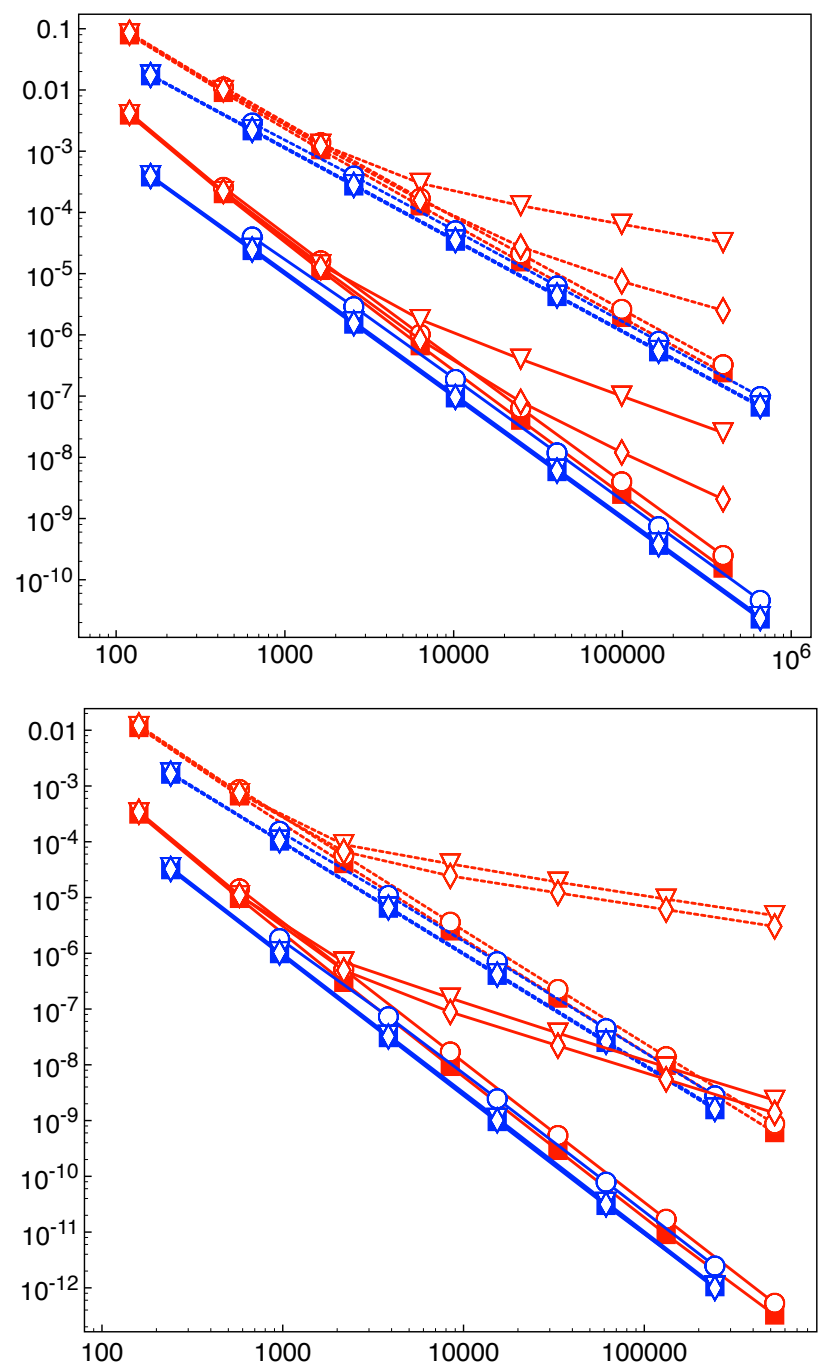

Figure 8: Error versus number of DOFs for HHO and DG discretizations of the Poisson equation over uniformly refined quadrilateral meshes. Forcing term and boundary conditions imposed according to the trigonometric solution 28 . 
- $\mathrm{HHO}$ cartesian quad4 $\mathrm{H} 1, \mathrm{POP} 1$ -O- $\mathrm{HHO}$ elemSubdiv quad8 $\mathrm{H} 1, \mathrm{POP} 1$ -- - - $\mathrm{HHO}$ randomDist quad8 $\mathrm{H1}$, POP1 $-\checkmark-\mathrm{HHO}$ regularDist quad8 $\mathrm{H1}$, P0P1

- dG cartesian quad4 $\mathrm{H} 1, \mathrm{P} 1$

- - dG elemSubdiv quad8 $\mathrm{H} 1, \mathrm{P} 1$

$-\nabla-\mathrm{dG}$ randomDist quad8 $\mathrm{H} 1, \mathrm{P} 1$

-- - dG regualrDist quad8 $\mathrm{H} 1, \mathrm{P} 1$

- HHO cartesian quad4 L2, POP1

$-\mathrm{O}-\mathrm{HHO}$ elemSubdiv quad8 $\mathrm{L} 2, \mathrm{POP}$

$-\nabla-\mathrm{HHO}$ randomDist quad8 $\mathrm{L} 2, \mathrm{POP}$

$\checkmark-\mathrm{HHO}$ regularDist quad8 $\mathrm{L} 2, \mathrm{POP} 1$

- dG cartesian quad8 L2, $P 1$

- dG elemSubdiv quad8 $L 2, P 1$

$-\nabla-\mathrm{dG}$ randomDist quad8 $\mathrm{L} 2, \mathrm{P} 1$

$\checkmark-d G$ regularDist quad8 $L 2, P 1$

$\mathrm{HHO}$ cartesian quad4 $\mathrm{H} 1, \mathrm{P} 1 \mathrm{P} 2$ - $\mathrm{HHO}$ elemSubdiv quad8 $\mathrm{H} 1, \mathrm{P} 1 \mathrm{P} 2$ $-\nabla-\mathrm{HHO}$ randomDist quad8 $\mathrm{H} 1, \mathrm{P} 1 \mathrm{P} 2$ - - $\mathrm{HHO}$ regularDist quad8 $\mathrm{H} 1, \mathrm{P} 1 \mathrm{P} 2$ - dG cartesian quad4 H1, P2 -O-dG elemSubdiv quad8 $\mathrm{H} 1, \mathrm{P} 2$ -- $\nabla-\mathrm{dG}$ randomDist quad8 $\mathrm{H1}, \mathrm{P} 2$

$-\checkmark-\mathrm{dG}$ regularDist quad8 $\mathrm{H} 1, \mathrm{P} 2$

- HHO cartesian quad4 L2, P1P2

- $-\mathrm{HHO}$ elemSubdiv quad8 L2, P1P2 $-\nabla-\mathrm{HHO}$ randomDist quad8 L2, P1P2 $\checkmark-\mathrm{HHO}$ regularDist quad8 L2, P1P2 - dG cartesian quad4 $L 2, P 2$

-O- dG elemSubdiv quad8 L2, P2

$-\nabla-\mathrm{dG}$ randomDist quad8 $\mathrm{L} 2, \mathrm{P} 2$

$\checkmark d \mathrm{dG}$ regularDist quad8 $\mathrm{L} 2, \mathrm{P} 2$
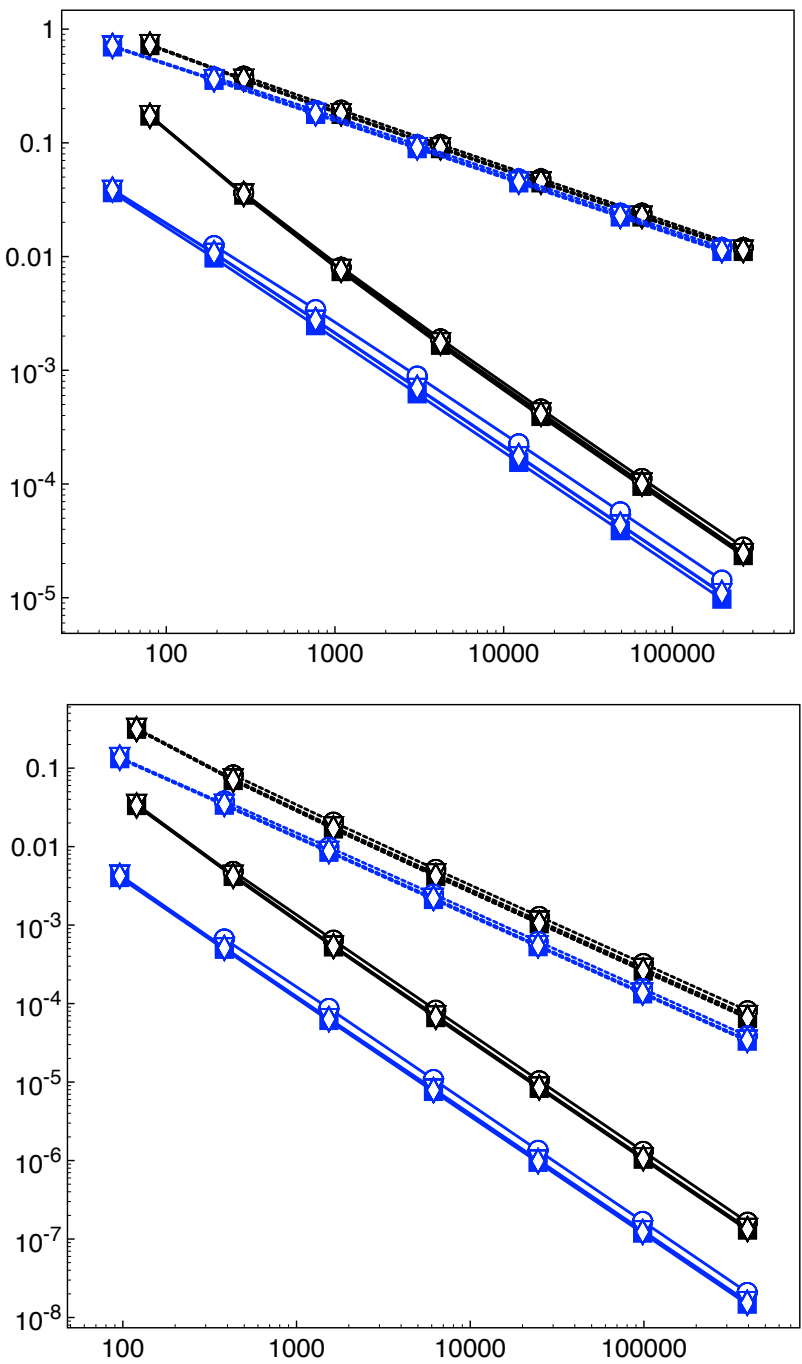

Figure 9: Error versus number of DOFs for HHO and DG discretizations of the Poisson equation over uniformly refined quadrilateral mesh sequences. Forcing term and boundary conditions imposed according to the trigonometric solution 28 . 
- $\mathrm{HHO}$ cartesian quad4 $\mathrm{H} 1, \mathrm{P} 2 \mathrm{P} 3$ -O- $\mathrm{HHO}$ elemSubdiv quad8 $\mathrm{H} 1, \mathrm{P} 2 \mathrm{P} 3$ $-\nabla-\mathrm{HHO}$ randomDist quad8 $\mathrm{H1}, \mathrm{P} 2 \mathrm{P} 3$ $--\mathrm{HHO}$ regularDist quad8 $\mathrm{H} 1, \mathrm{P} 2 \mathrm{P} 3$

- dG cartesian quad4 $\mathrm{H} 1, \mathrm{P} 3$ -O-dG elemSubdiv quad8 $\mathrm{H} 1, \mathrm{P} 3$ - $\nabla-\mathrm{dG}$ randomDist quad8 $\mathrm{H1}, \mathrm{P3}$ $-\checkmark-\mathrm{dG}$ regularDist quad8 $\mathrm{H} 1, \mathrm{P} 3$ - $\mathrm{HHO}$ cartesian quad4 L2, P2P3 $-\mathrm{O}-\mathrm{HHO}$ elemSubdiv quad8 L2, P2P3 $-\nabla-\mathrm{HHO}$ randomDist quad8 L2, P2P3 $\checkmark-\mathrm{HHO}$ regularDist quad8 $\mathrm{L} 2, \mathrm{P} 2 \mathrm{P} 3$

- dG cartesian quad4 L2, P3 - $-d G$ elemSubdiv quad8 $L 2, P 3$ $-\nabla-\mathrm{dG}$ randomDist quad8 $\mathrm{L} 2, \mathrm{P} 3$ $\checkmark d G$ regularDist quad8 $L 2, P 3$
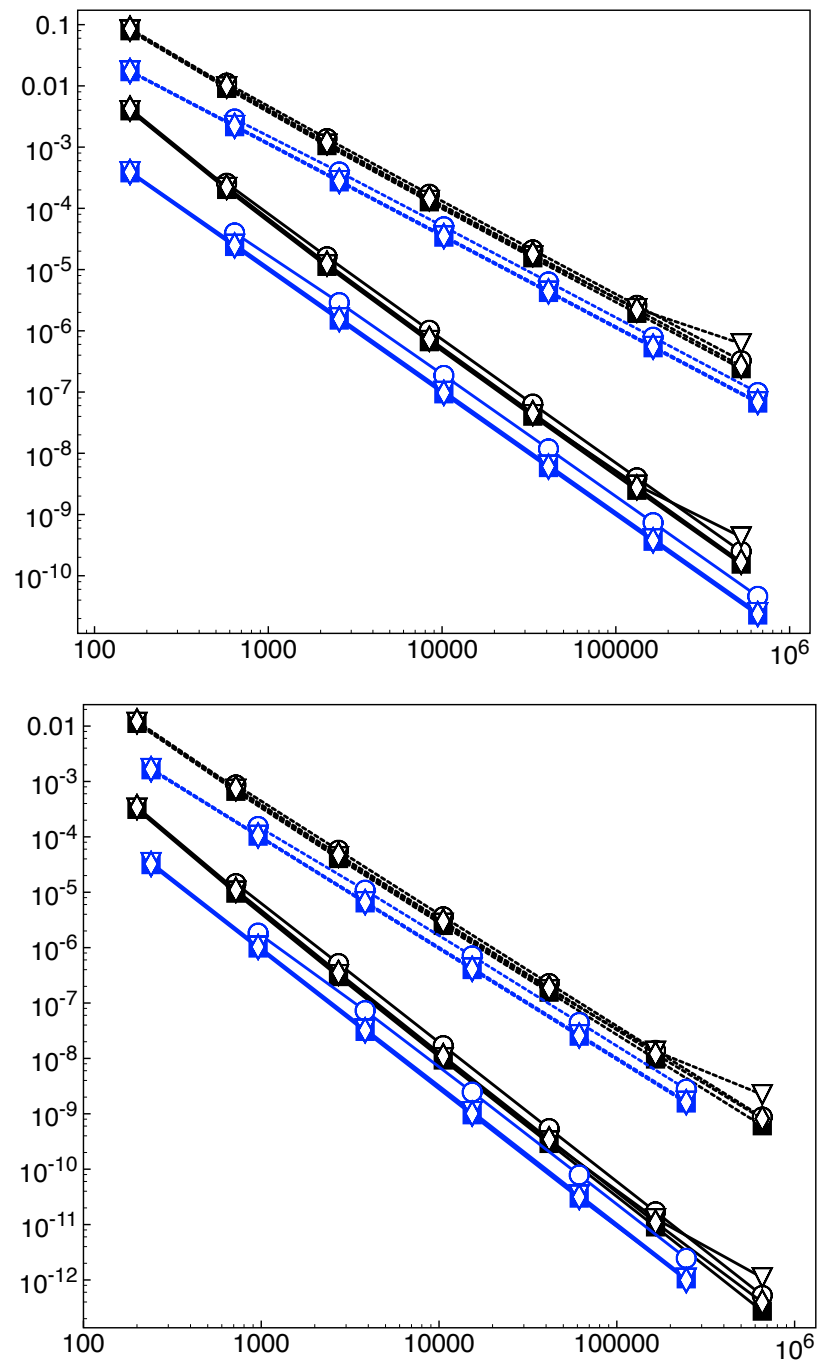

Figure 10: Error versus number of DOFs for HHO and DG discretizations of the Poisson equation over uniformly refined quadrilateral mesh sequences. Forcing term and boundary conditions imposed according to the trigonometric solution 28 . 
- $\mathrm{HHO}$ regular tet4 $\mathrm{H} 1, \mathrm{POP} 1$ $\diamond \mathrm{HHO}$ randomDist tet10 H1, POP1

- $\mathrm{HHO}$ regular tet $4 \mathrm{H} 1, \mathrm{POPO}$

$\diamond \mathrm{HHO}$ randomDist tet $10 \mathrm{H} 1, \mathrm{POPO}$

- dG regular tet $4 \mathrm{H} 1, \mathrm{P} 1$

$\diamond \mathrm{dG}$ randomDist tet $10 \mathrm{H} 1, \mathrm{P} 1$

- HHO regular tet4 L2, POP1

$\checkmark-$ HHO randomDist tet10 L2, POP1

- $\mathrm{HHO}$ regular tet4 $\mathrm{L} 2, \mathrm{POPO}$

$\checkmark-$ HHO randomDist tet10 L2, POPO

- dG regular tet4 $\mathrm{L} 2, \mathrm{P1}$

$\neg-d G$ randomDist tet10 L2, P1

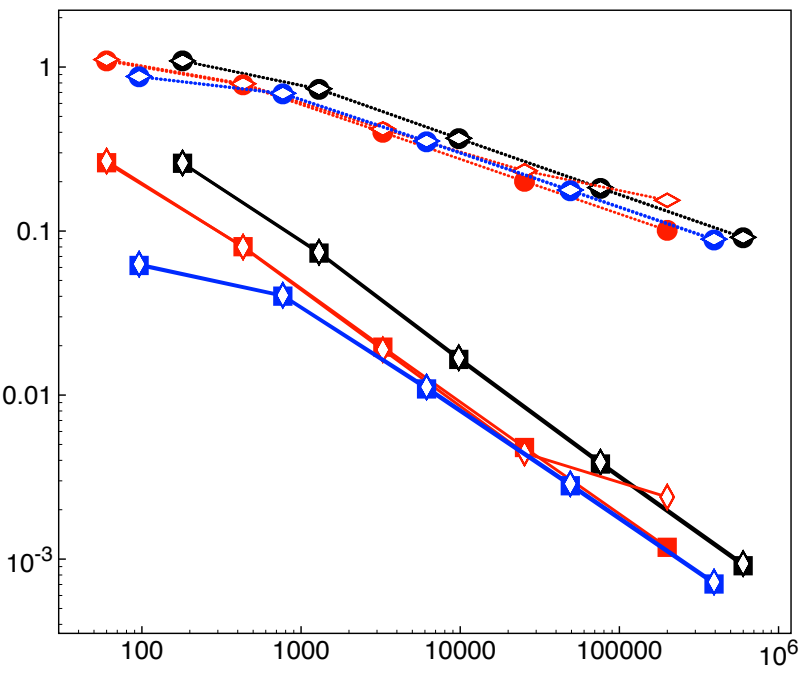

HHO regular tet4 $\mathrm{H} 1, \mathrm{P} 1 \mathrm{P} 2$ HHO randomDist tet10 H1, P1P2

- $\mathrm{HHO}$ regular tet $4 \mathrm{H} 1, \mathrm{P} 1 \mathrm{P} 1$

$\checkmark$ HHO randomDist tet10 H1, P1P1

- dG regular tet4 $\mathrm{H} 1, \mathrm{P} 2$

$\diamond \mathrm{dG}$ randomDist tet $10 \mathrm{H} 1, \mathrm{P} 2$

- $\mathrm{HHO}$ regular tet4 L2, P1P2

$\checkmark-\mathrm{HHO}$ randomDist tet10 L2, P1P2

$-\mathrm{HHO}$ regular tet4 $\mathrm{L} 2, \mathrm{P} 1 \mathrm{P} 1$

$-\checkmark-\mathrm{HHO}$ randomDist tet10 L2, P1P1

$-\mathrm{dG}$ regular tet4 $\mathrm{L} 2, \mathrm{P} 2$

$-\checkmark-d G$ randomDist tet10 L2, P2

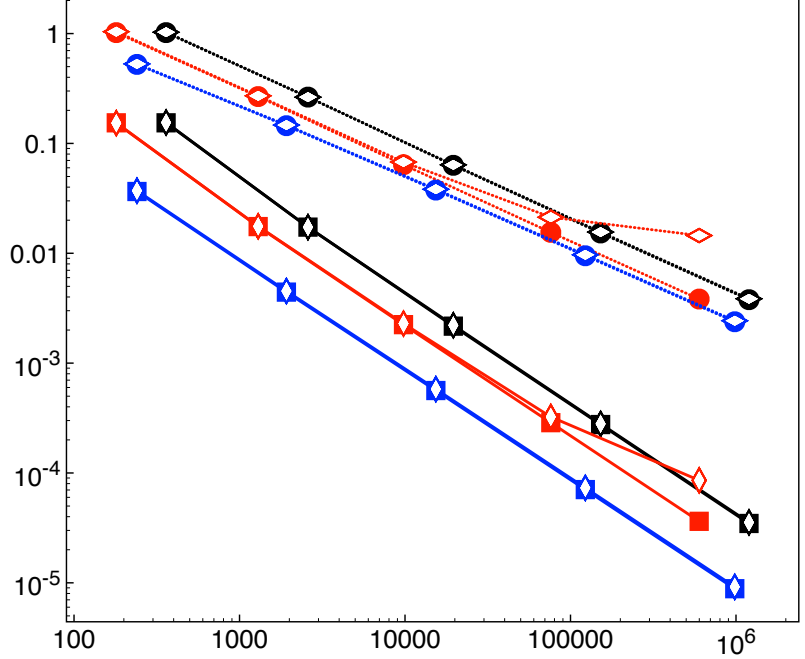

Figure 11: Error versus number of DOFs for HHO and DG discretizations of the Poisson equation over uniformly refined tetrahedral mesh sequences. Forcing term and boundary conditions imposed according to the trigonometric solution 28. 
- $\mathrm{HHO}$ regular tet4 $\mathrm{H} 1, \mathrm{P} 2 \mathrm{P} 3$

$\diamond-\mathrm{HHO}$ randomDist tet10 H1, P2P3

- $\mathrm{HHO}$ regular tet4 $\mathrm{H} 1, \mathrm{P} 2 \mathrm{P} 2$

$\diamond-\mathrm{HHO}$ randomDist tet10 H1, P2P2

- dG regular tet4 $\mathrm{H} 1, \mathrm{P} 3$

$\diamond$ dG randomDist tet10 $\mathrm{H} 1, \mathrm{P3}$

- $\mathrm{HHO}$ regular tet4 $\mathrm{L} 2, \mathrm{P} 2 \mathrm{P} 3$

$\checkmark-\mathrm{HHO}$ randomDist tet10 L2, P2P3

$-\mathrm{HHO}$ regular tet4 L2, P2P2

$\checkmark-\mathrm{HHO}$ randomDist tet10 L2, P2P2

- dG regular tet4 $\mathrm{L} 2, \mathrm{P} 3$

$\checkmark$ dG randomDist tet10 L2, P3
- $\mathrm{HHO}$ regular tet4 $\mathrm{H} 1, \mathrm{P} 3 \mathrm{P} 4$ HHO randomDist tet10 $\mathrm{H} 1, \mathrm{P} 3 \mathrm{P} 4$

- $\mathrm{HHO}$ regular tet4 $\mathrm{H} 1, \mathrm{P} 3 \mathrm{P} 3$

$\diamond$ HHO randomDist tet10 H1, P3P3

- dG regular tet4 $\mathrm{H} 1, \mathrm{P} 4$

$\diamond \mathrm{dG}$ randomDist tet $10 \mathrm{H} 1, \mathrm{P} 4$

- $\mathrm{HHO}$ regular tet4 L2, P3P4

$\checkmark \mathrm{HHO}$ randomDist tet10 L2, P3P4

$-\mathrm{HHO}$ regular tet4 $\mathrm{L} 2, \mathrm{P} 3 \mathrm{P} 3$

$\checkmark-\mathrm{HHO}$ randomDist tet10 L2, P3P3

$-\mathrm{dG}$ regular tet4 $\mathrm{L} 2, \mathrm{P} 4$

$\checkmark-d G$ randomDist tet10 L2, P4
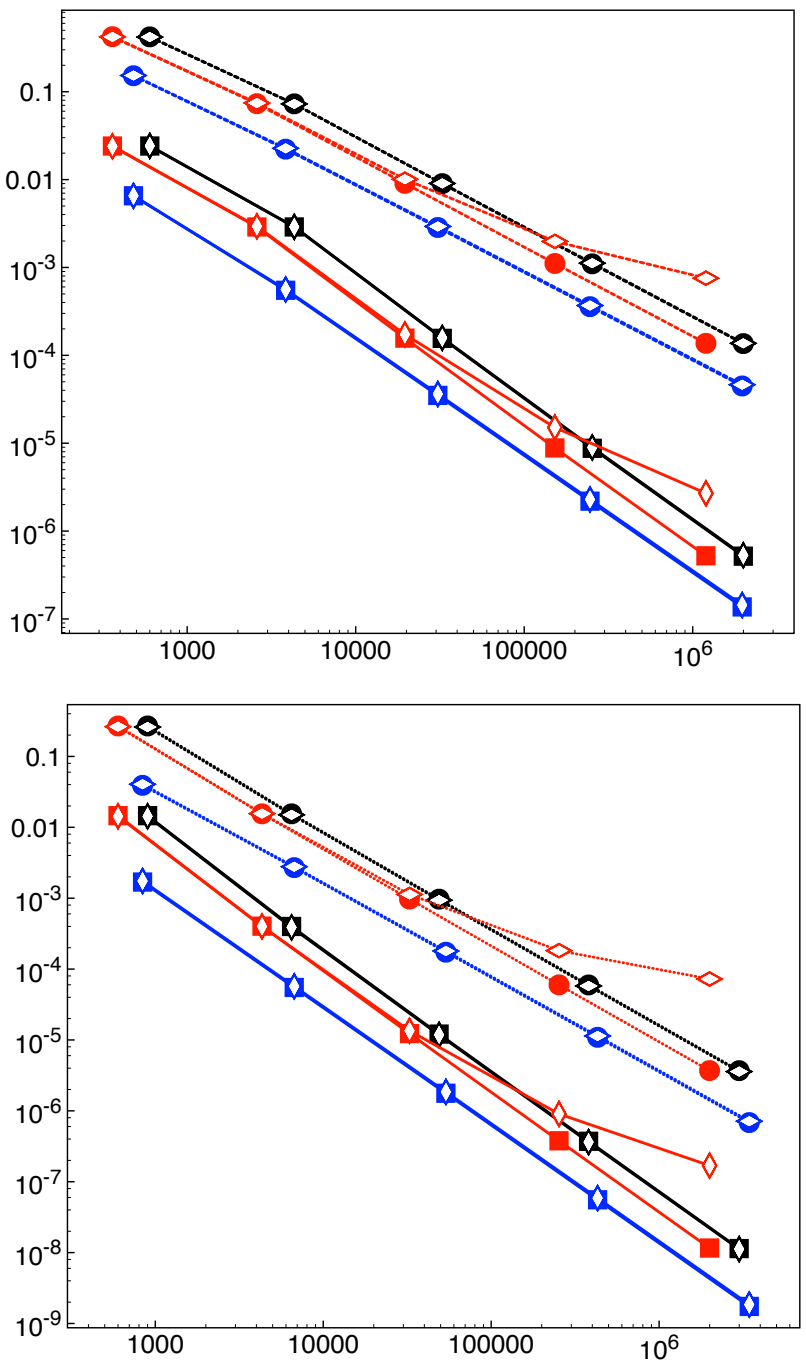

Figure 12: Error versus number of DOFs for HHO and DG discretizations of the Poisson equation over uniformly refined tetrahedral mesh sequences. Forcing term and boundary conditions imposed according to the trigonometric solution 28 . 
- HHO elemSub hex20 H1, P0P1 $\checkmark$ HHO randomDist hex20 H1, P0P1 - HHO elemSub hex $20 \mathrm{H} 1, \mathrm{POPO}$

$\diamond$ HHO randomDist hex20 H1, P0P0

- dG elemSub hex20 H1, P1

$\diamond \mathrm{dG}$ randomDist hex20 $\mathrm{H} 1, \mathrm{P} 1$

- $\mathrm{HHO}$ elemSub hex20 L2, P0P1

$\checkmark-\mathrm{HHO}$ randomDist hex20 L2, P0P1

- HHO elemSub hex20 L2, POPO

$-\downarrow \mathrm{HHO}$ randomDist hex20 L2, POPO

- dG elemSub hex20 L2, P1

$\checkmark-d G$ randomDist hex20 L2, P1
- HHO elemSub hex20 H1, P1P2 $\checkmark$ HHO randomDist hex20 H1, P1P2

- HHO elemSub hex20 H1, P1P1

$\diamond \mathrm{HHO}$ randomDist hex20 $\mathrm{H} 1, \mathrm{P} 1 \mathrm{P} 1$

- dG elemSub hex20 H1, P2

$\diamond \mathrm{dG}$ randomDist hex20 $\mathrm{H} 1, \mathrm{P} 2$

- HHO elemSub hex20 L2, P1P2

$\checkmark$ - HHO randomDist hex20 L2, P1P2

- HHO elemSub hex20 L2, P1P1

$\checkmark-\mathrm{HHO}$ randomDist hex20 L2, P1P1

$-d G$ elemSub hex20 L2, P2

$\checkmark$ dG randomDist hex20 L2, P2
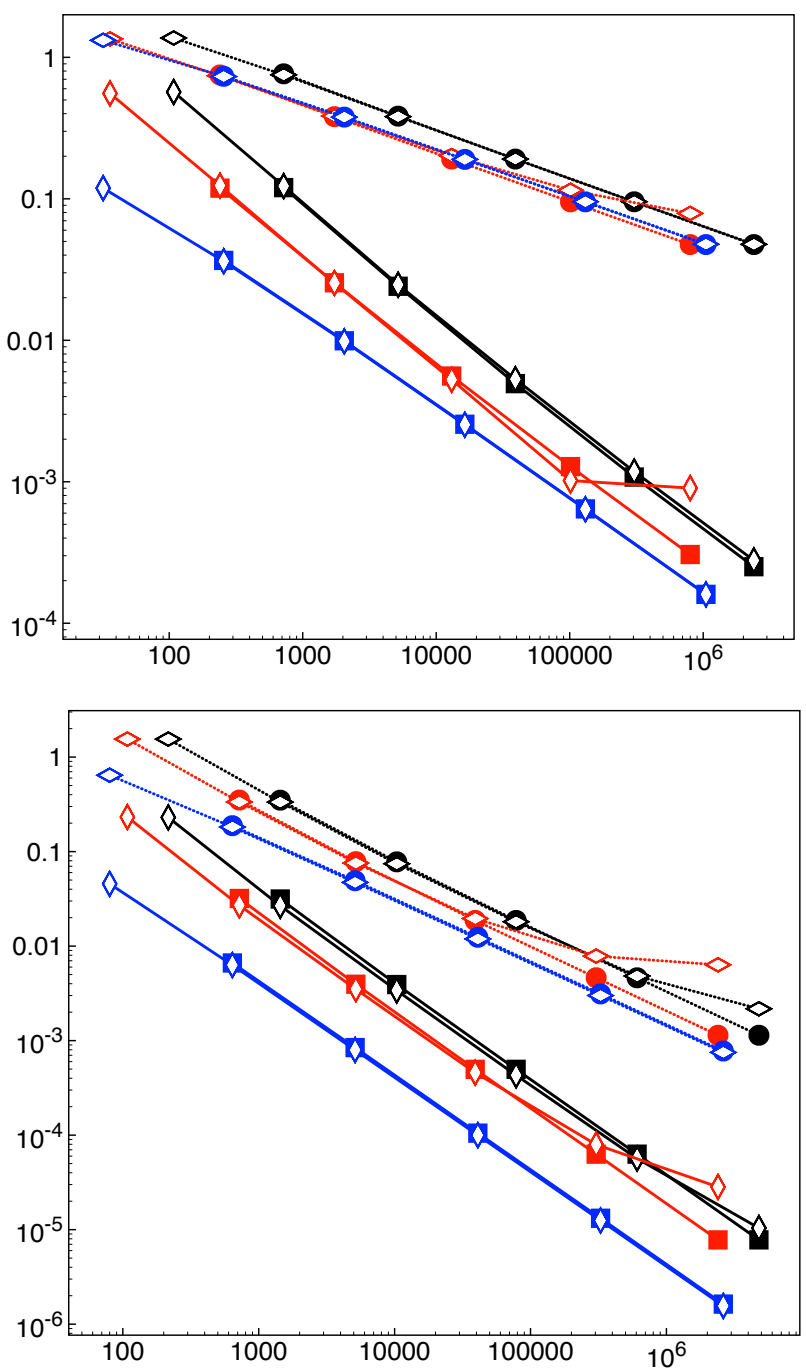

Figure 13: Error versus number of DOFs for HHO and DG discretizations of the Poisson equation over uniformly refined hexahedral mesh sequences. Forcing term and boundary conditions imposed according to the trigonometric solution 28 . 
- $\mathrm{HHO}$ elemSub hex20 H1, P2P3 $\diamond \mathrm{HHO}$ randomDist hex20 H1, P2P3

- $\mathrm{HHO}$ elemSub hex20 H1, P2P2

$\diamond \mathrm{HHO}$ randomDist hex20 H1, P2P2

- dG elemSub hex20 H1, P3

$\diamond \mathrm{dG}$ randomDist hex20 H1, P3

- $\mathrm{HHO}$ elemSub hex20 L2, P2P3

$\checkmark-\mathrm{HHO}$ randomDist hex20 L2, P2P3

$-\mathrm{HHO}$ elemSub hex20 L2, P2P2

$\checkmark-\mathrm{HHO}$ randomDist hex20 L2, P2P2

- dG elemSub hex20 L2, P3

$\checkmark-d G$ randomDist hex20 L2, P3
- HHO elemSub hex20 H1, P3P4 $\checkmark$. $\mathrm{HHO}$ randomDist hex20 H1, P3P4

- HHO elemSub hex20 H1, P3P3

$\diamond \mathrm{HHO}$ randomDist hex20 H1, P3P3

- dG elemSub hex20 H1, P4

$\diamond \mathrm{dG}$ randomDist hex20 $\mathrm{H} 1, \mathrm{P} 4$

- HHO elemSub hex20 L2, P3P4

$\checkmark-\mathrm{HHO}$ randomDist hex20 L2, P3P4

- - HHO elemSub hex20 L2, P3P3

$\checkmark$ - HHO randomDist hex20 L2, P3P3

dG elemSub hex20 L2, P4

$\checkmark \mathrm{dG}$ randomDist hex20 L2, P4
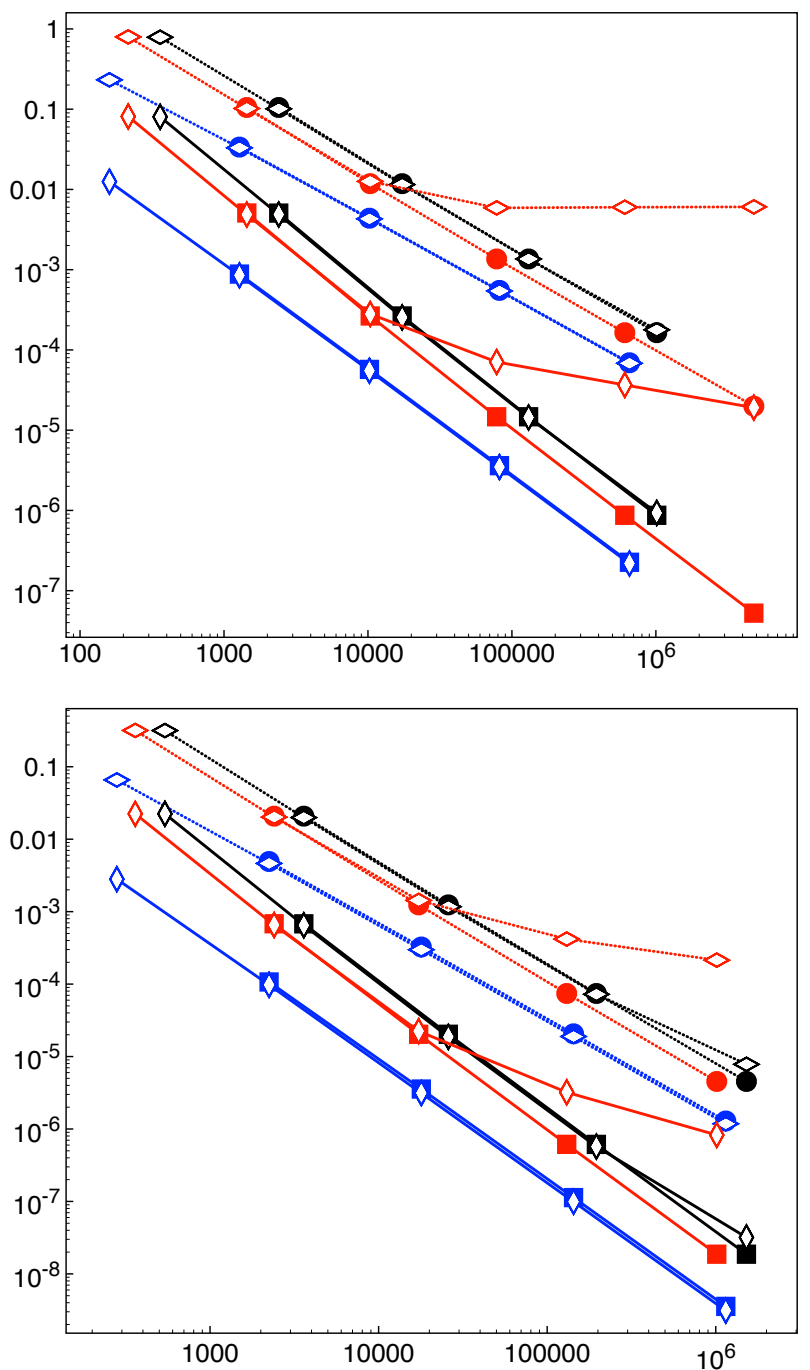

Figure 14: Error versus number of DOFs for HHO and DG discretizations of the Poisson equation over uniformly refined hexahedral mesh sequences. Forcing term and boundary conditions imposed according to the trigonometric solution 28 . 

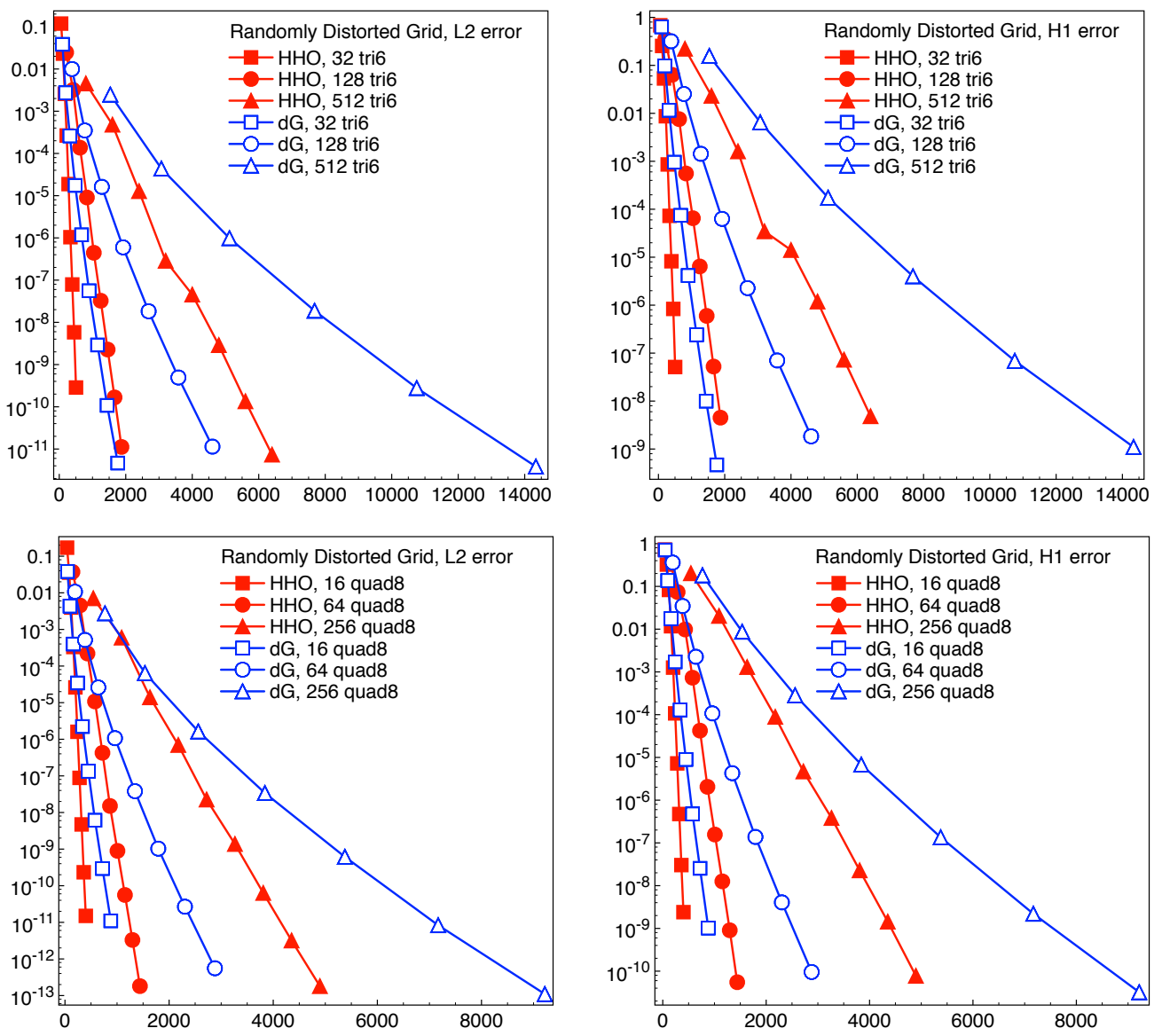

Figure 15: Error versus number of DOFs. $p$-refined HHO and DG discretizations of the Poisson equation over the six-node distorted triangular meshes of Figure 1a (first row) and the eight-node distorted quadrilateral meshes of Figure $1 \mathrm{~b}$ (second row). HHO DOFs space is $\underline{\mathrm{U}}_{h}^{k, k}(k=0,1, \ldots)$, DG DOFs space is $\mathrm{U}_{h}^{k}(k=1,2, \ldots)$. 

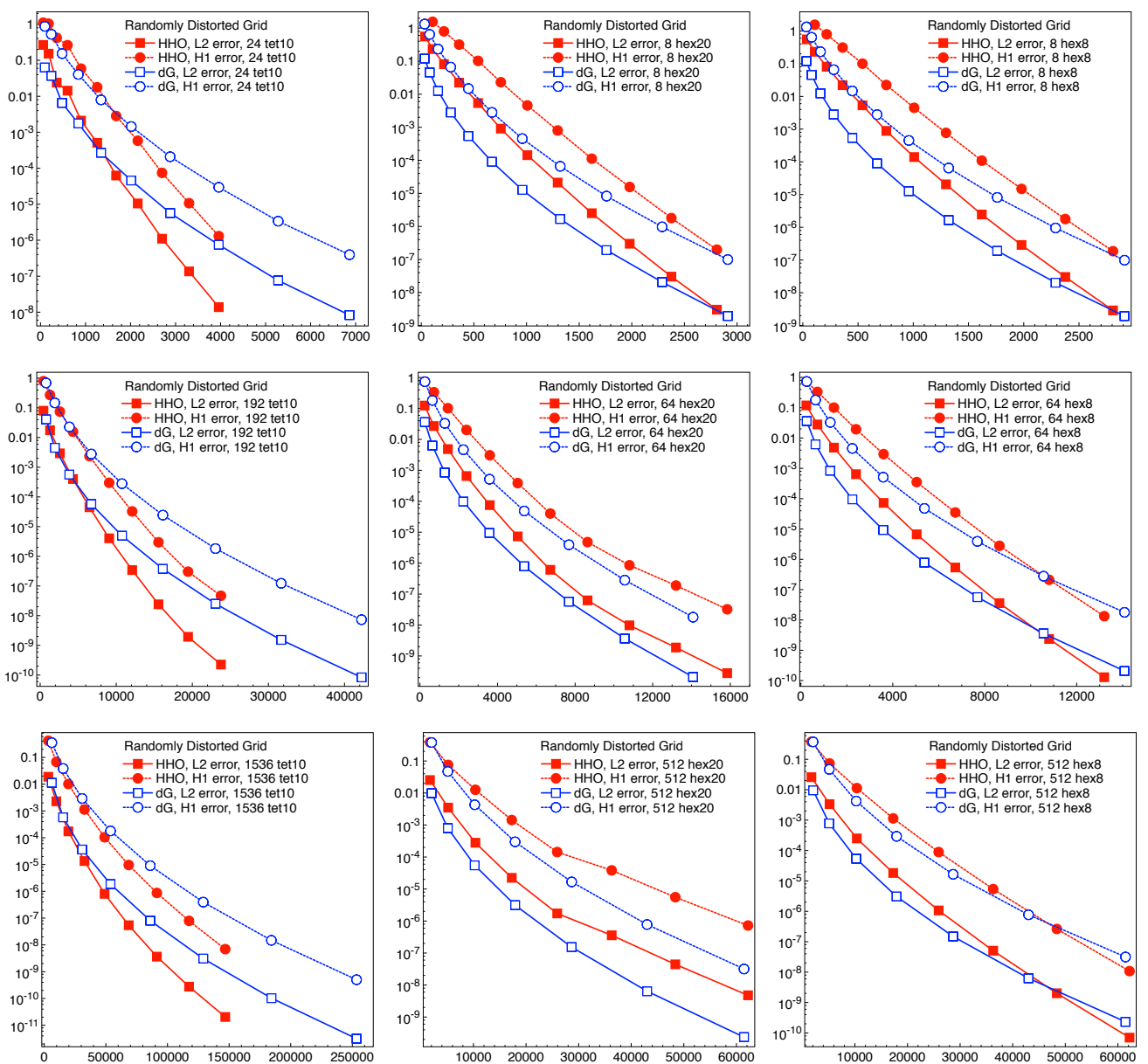

Figure 16: Error versus number of DOFs. p-refined HHO and DG discretizations of the Poisson equation over the ten-node distorted tetrahedral meshes of Figure 2a (first column), the twenty-node distorted hexahedral meshes of Figure 2c (second column) and and the eight-node distorted hexahedral meshes of Figure $2 \mathrm{~b}$ (third column). HHO DOFs space is $\underline{\mathrm{U}}_{h}^{k, k}(k=0,1, \ldots)$, DG DOFs space is $\mathrm{U}_{h}^{k}(k=1,2, \ldots)$. 
major advantage of high-order discretizations. In this section we consider the same trigonometric solution (28) as in the previous section, but we focus on $p$-convergence instead of $h$-convergence. In this context, HHO discretizations can be expected to provide significant gains with respect to (modal) DG discretizations, since the corresponding number of DOFs grows significantly slower than for DG methods when increasing the polynomial degree.

The numerical results reported in Figures 15 and 16 for two-dimensional randomly distorted triangular and quadrilateral meshes, and three-dimensional randomly distorted hexahedral and tetrahedral meshes, respectively, confirm this trend in all but the hexahedral meshes. In particular, the randomly distorted twenty-node hexahedral meshes seem more favorable to $p$-refined DG discretizations rather than HHO discretizations. This behaviour can be explained taking into account the following aspects:

- For the DG discretizations, we have chosen the smallest possible polynomial space $\mathbb{P}_{d}^{k}(T)$ over each $T \in \mathcal{T}_{h}$, irrespectively of the element shape.

- For HHO discretizations, the global number of DOFs is proportional to the number of faces, and hexahedral elements have an unfavourable number of faces per element compared to tetrahedra.

- The effective mapping order $\mathrm{m}$ is higher for eight-node quadrilateral faces $(\mathrm{m}=3)$, as compared to six-node triangular mesh faces and four-node quadrilateral mesh faces $(m=2)$. Accordingly, the convergence degradation of reference frame polynomial spaces $\mathbb{P}_{d-1}^{l}(\sigma)$ is the most severe of the lot, and requires higher polynomial degrees on faces to compensate.

We also notice that we have placed ourselves in the worst-case scenario, where all the faces are curved. If curved faces only occur on the boundary of the domain, a significant reduction in the number of DOFs can be obtained for HHO using equal element and face polynomial orders for the internal faces.

\section{Agglomeration coarsening}

Besides the advantage in terms of accuracy per DOF, one of the major benefits of $p$-convergence with respect to $h$-convergence is the fact that the former is a single grid strategy, while the latter requires to generate a $h$-refined mesh sequence. Clearly, in order to keep the global number of DOFs under control and fruitfully exploit high polynomial degrees, a grid conceived for $p$-refinement must be coarse enough. On the other hand, when considering a complex domain $\Omega$, the mesh must also meet the conflicting requirement of providing a satisfactory approximation $\Omega_{h}$ of the domain: a rough approximation can be highly detrimental in terms of accuracy. In particular, the enforcement of boundary conditions on $\partial \Omega_{h}$ might lead to large consistency errors that are not settled increasing the polynomial degree $k$.

Unfortunately, generating a coarse high-order grid $\mathcal{T}_{h}$ that provides a satisfactory representation of the domain boundary $\partial \Omega$ is often a non-trivial task. In practice, building a $h$-refined mesh sequence might be both less expensive and time consuming. Moreover, $h$-refined mesh sequences can be constructed in such a way that $\Omega_{h}$ is a better and better approximation of $\Omega$ as $h$ decreases. 
In this context, the support of arbitrarily shaped elements in HHO and DG discretizations comes into play, providing an unprecedented flexibility by means of agglomeration coarsening. Starting from a fine first- or second-order grid $\mathcal{T}_{h}$, an agglomerated grid $\mathcal{T}_{H}$ can be generated on the fly by clustering together the cells of the fine grid, as detailed in Section 6.1 below. The grid $\mathcal{T}_{H}$ can be made arbitrarily coarse, so that $\operatorname{card}\left(\mathcal{T}_{H}\right) \ll \operatorname{card}\left(\mathcal{T}_{h}\right)$, while keeping the approximation of the computational domain unchanged, i.e., $\Omega_{H}=\Omega_{h}$.

\subsection{Agglomerated mesh}

Starting from a mesh $\mathcal{T}_{h}$, we can define a coarsened mesh $\mathcal{T}_{H}=\{\mathfrak{T}\}$ by agglomeration. More precisely, we suppose that (i) $\mathcal{T}_{H}$ partitions $\Omega_{h}:=\bigcup_{T \in \mathcal{T}_{h}} \bar{T} \backslash \partial \Omega$ in the sense that $\bigcup_{\mathfrak{T} \in \mathcal{T}_{H}} \overline{\mathfrak{T}}=\overline{\Omega_{h}}$; (ii) every agglomerated element $\mathfrak{T} \in \mathcal{T}_{H}$ is an open bounded connected subset of $\Omega$ and there exists a set of sub-elements $\mathcal{T}_{\mathfrak{T}} \subset \mathcal{T}_{h}$ such that

$$
\overline{\mathfrak{T}}=\bigcup_{T \in \mathcal{T}_{\mathfrak{T}}} \bar{T}
$$

We define a facet $F$ of $\mathfrak{T} \in \mathcal{T}_{H}$ as a portion of its boundary $\partial \mathfrak{T}$ such that $F \in \mathcal{F}_{h}$. Facets are collected in the set $\mathcal{F}_{h}^{H} \subset \mathcal{F}_{h}$. By definition, for every facet there exists a reference face $\sigma \in \mathcal{K}^{d-1}$ and a polynomial mapping $\boldsymbol{\Psi}_{F}$ such that $F=\boldsymbol{\Psi}_{F}(\sigma)$. We define an agglomerated face $\mathfrak{F}$ of $\mathfrak{T} \in \mathcal{T}_{H}$ as a portion of $\partial \mathfrak{T}$ such that either $\mathfrak{F}=\partial \mathfrak{T} \cap \partial \Omega$ or there exists $\mathfrak{T}^{\prime} \in \mathcal{T}_{H}, \mathfrak{T}^{\prime} \neq \mathfrak{T}$, such that $\mathfrak{F}=\partial \mathfrak{T} \cap \partial \mathfrak{T}^{\prime}$. Agglomerated faces are collected in the set $\mathcal{F}_{H}$. For every agglomerated face $\mathfrak{F} \in \mathcal{F}_{H}$ we introduce the set $\mathcal{F}_{\mathfrak{F}} \subset \mathcal{F}_{h}^{H}$ collecting the facets partitioning $\mathfrak{F}$, i.e., such that $\overline{\mathfrak{F}}=\bigcup_{F \in \mathcal{F}_{\mathfrak{F}}} \bar{F}$.

Both HHO and DG discretizations can be extended to support agglomerated meshes pending the following changes:

- For both $H H O$ and $D G$ discretizations: $\mathcal{T}_{h}$ is replaced by $\mathcal{T}_{H}$ and, correspondingly, $T \in \mathcal{T}_{h}$ is replaced by $\mathfrak{T} \in \mathcal{T}_{H}$.

- For DG discretizations only: $\mathcal{F}_{h}$ is replaced by $\mathcal{F}_{H}$ and, correspondingly, $F \in \mathcal{F}_{h}$ is replaced by $\mathfrak{F} \in \mathcal{F}_{H}$.

- For HHO discretizations only: $\mathcal{F}_{h}$ is replaced by $\mathcal{F}_{h}^{H}$.

\subsection{Numerical results}

In what follows, we use agglomeration coarsening to demonstrate that $p$-convergence can be fruitfully exploited to reduce the error with respect to exact solution up to a tight tolerance. Most importantly, this can be achieved starting from a single standard fixed fine mesh $\mathcal{T}_{h}$ of a curved computational domain. To assess the $p$-convergence rates of the DG and HHO methods we consider the test case of Gobbert and Yang [37]: the computational domain is is unit annulus $\Omega=\left\{0.5<x^{2}+y^{2}<1.5\right\}$ and we consider the following exact solution of the Poisson equation:

$$
u(\mathbf{x})=\cos \left(\pi \sqrt{x^{2}+y^{2}}\right),
$$


with suitable forcing term $f$. The solution 29) vanishes on the exact boundary $\partial \Omega$. We consider second-order six-node triangular mesh sequences and the following discretizations of the homogeneous boundary condition:

$$
\begin{array}{ll}
\text { Choice 1: } & \left.u(\mathbf{x})\right|_{\partial \Omega_{h}}=\cos \left(\pi \sqrt{x^{2}+y^{2}}\right), \\
\text { Choice 2: } & \left.u(\mathbf{x})\right|_{\partial \Omega_{h}}=0 .
\end{array}
$$

The choice 30a neglects any influence of the domain discretization as we are using the exact solution on $\partial \Omega_{h}$; instead, the choice $30 \mathrm{~b}$ introduces a consistency error since, in our case, $\partial \Omega_{h} \neq \partial \Omega$.

The agglomerated and regular $h$-coarsened mesh sequences are built as follows:

- Agglomerated mesh sequence (Figure 17a): we agglomerate on top of a fine 40k six-node triangular elements grid $\mathcal{T}_{h}$. Starting from a $4 \mathrm{k}$ agglomerated elements grid $\mathcal{T}_{H_{1}}$ (obtained by agglomerating ten fine grid elements on average), for $i \in$ $\{2, \ldots, 8\}$, we construct agglomerated meshes such that $\operatorname{card}\left(\mathcal{T}_{H_{i}}\right)=\operatorname{card}\left(\mathcal{T}_{H_{i-1}}\right)\left(\frac{\operatorname{dim}\left(\mathbb{P}_{d}^{i-1}\right)}{\operatorname{dim}\left(\mathbb{P}_{d}^{i}\right)}\right)$.

- Regular mesh sequence (Figure 17b): we mimick the mesh cardinality of the agglomerated mesh sequence, that is $\operatorname{card}\left(\mathcal{T}_{h_{i}}\right) \approx \operatorname{card}\left(\mathcal{T}_{H_{i}}\right)$, such that $h_{i} \approx H_{i}$.

Note that the discrete computational domain $\Omega_{H_{i}}=\Omega_{h}$ is fixed when we consider the agglomerated mesh sequence, depending solely on the underlying grid $\mathcal{T}_{h}$. As opposite, coarser regular meshes provide a rougher approximation of the domain boundaries $\partial \Omega_{h_{i}}$ when compared to finer regular meshes.

Non-conforming HHO and DG discretizations are considered over agglomerated and regular mesh sequences using both boundary condition choices in (30).

- $D G$ : we consider the spaces $\mathrm{U}_{H_{i}}^{i}$ and $\mathrm{U}_{h_{i}}^{i}$, the resulting DG discretizations have approximatively $12 \mathrm{k}$ DOFs.

- HHO: we consider equal-order HHO discretizations based of the spaces $\underline{\mathrm{U}}_{H_{i}}^{i-1, i-1}$ and $\underline{\mathrm{U}}_{h_{i}}^{i-1, i-1}$. Note that degree $k \mathrm{DG}$ and degree $k-1 \mathrm{HHO}$ discretizations provide the same $h$-convergence rates.

The convergence results shown in Table 5 and Figure 18 demonstrate the ability of approaching (up to $10^{-9}$ ) the exponential convergence results obtained enforcing the exact boundary condition (30a) using agglomerated mesh sequences together with the boundary condition (30b). Instead, the lack of consistency for the regular mesh sequence is clearly appreciable as the discretization is unable to provide an error in the $L^{2}$-norm lower that $10^{-5}$ using the boundary condition in (30b).

It is interesting to remark that DG solutions based on the boundary condition in (30a) provide comparable results over the agglomerated and regular mesh sequence since the number of DOFs is proportional to the number of mesh elements. As opposite, HHO discretizations based on the boundary condition in 30a perform better on the agglomerated grid but also require a significantly higher number of DOFs, see Table 5. This is due to the fact that agglomerated elements have a significantly higher number of facets as compared to triangular elements, hence $\operatorname{card}\left(\mathcal{F}_{h}^{H}\right) \gg \operatorname{card}\left(\mathcal{F}_{h}\right)$ even if $\operatorname{card}\left(\mathcal{T}_{H}\right) \approx \operatorname{card}\left(\mathcal{T}_{h}\right)$. 
Table 5 also reports the number of ILU preconditioned Conjugate Gradient iterations required to reduce the relative residual norm of the global equation system by fourteen orders of magnitude. For both the HHO and dG methods, the performance of the iterative solver is not affected by the use of agglomerated meshes. This behaviour is expected for $\mathrm{dG}$ methods, where the number of mesh faces on agglomerated meshes is comparable to standard meshes (since faces are defined as intersections of two elements or of one element with the domain boundary, see [23, Definition 1.16]). Interestingly, a similar behaviour is observed also for HHO methods, where the faces of the agglomerated mesh coincide with facets; see 31 for further details on the notion of mesh faces for HHO. Furthermore, increasing the polynomial degree on coarser agglomerated meshes is highly beneficial from both the accuracy and the easiness of solution viewpoints.

We conclude this section by observing that the main issue of mesh agglomeration is related to numerical integration. As a matter of fact, if quadrature is performed on the subelements, as we actually do, the cost of numerical integration can become significant.

\section{Conclusion}

We assessed the convergence rates of a new adaptation of the HHO method to curved meshes based on physical frame polynomial spaces over mesh elements and reference frame polynomial spaces over mesh faces. We verified, by means of carefully crafted numerical test cases, that optimal approximation properties of the potential reconstruction operator can be recovered by increasing the degree of face polynomial spaces by a suitable amount with respect to element polynomial spaces. This amount can be theoretically estimated according to the face shape and the mapping properties so as to counterbalance the convergence degradation affecting reference frame polynomials in case of non-linear mappings.

We demonstrated (and motivated by analyzing the error of the reconstructed potential with respect to the exact solution) that optimal convergence rates can be expected on non-pathological mesh sequences for standard HHO approximations using the same polynomial degree on both elements and faces. By non-pathological we mean here that they tend towards an affine mesh when the meshsize is reduced. On the other hand, when dealing with pathological mesh sequences (which do not tend to affine meshes upon refinement), the numerical evidence shows that most of the time sufficient accuracy can be obtained by increasing the face polynomial degree by 1 . Clearly, non-pathological mesh sequences are representative of most practical applications thanks to the availability of good mesh generators, see e.g. [38. As opposite, handling pathological mesh sequences may be relevant in the context of moving and deformable mesh methods. We remark that, both in pathological and non-pathological mesh sequences, we considered the worst possible configuration where all the elements are curved, not only the elements that are adjacent to domain boundaries.

The comparison of HHO and DG methods with the same convergence rates in $h$ shows that the errors in both the $L^{2}$ - and $H^{1}$-norms are comparable, with a small but perceptible advantage of DG in terms of accuracy per DOF for polynomial degrees greater than one. This behavior is documented comparing (i) HHO discretization based on the equal-order space $\underline{\mathrm{U}}_{h}^{k, k}$ HHO with DG discretization based on the space $\mathrm{U}_{h}^{k+1}$ over nonpathological mesh sequences and (ii) HHO discretizations based on the enriched space $\underline{\mathrm{U}}_{h}^{k, k+1}$ with DG based on the space $\mathrm{U}_{h}^{k+1}$ over pathological mesh sequences. 
Table 5: $p$-convergence of HHO and DG discretizations on $h$-coarsened agglomerated and regular mesh sequence of Figures $17 \mathrm{a} \cdot 17 \mathrm{~b} L^{2}$ and $H^{1}$ measure the error in $L^{2}\left(\Omega_{h}\right)$ norm with respect to the exact solution and the exact solution gradient, respectively, using the boundary condition in 30a and 30b $\left(\operatorname{ex}_{\mathrm{BC}}\right.$ and ho $\mathrm{BC}$, respectively).. The last column shows the number of iterations of the linear solver (ILU preconditioned Conjugate Gradient).

\begin{tabular}{|c|c|c|c|c|c|c|c|c|}
\hline$k$ & $\operatorname{card}\left(\mathcal{T}_{h}\right)$ & $\operatorname{card}\left(\mathcal{F}_{h}\right)$ & DOFs & $L^{2} \mathrm{ex}_{\mathrm{BC}}$ & $L^{2} \mathrm{ho}_{\mathrm{BC}}$ & $H^{1} \operatorname{ex}_{\mathrm{BC}}$ & $H^{1}$ hовC $_{\text {B }}$ & LS its \\
\hline \multicolumn{9}{|c|}{ DG discretizations on agglomerated meshes $\left(\mathcal{T}_{h}=\mathcal{T}_{H}, \mathcal{F}_{h}=\mathcal{F}_{H}\right)$} \\
\hline 1 & 4080 & 11683 & 12240 & $3.04 \mathrm{e}-03$ & $3.04 \mathrm{e}-03$ & $2.32 \mathrm{e}-01$ & $2.32 \mathrm{e}-01$ & 169 \\
\hline 2 & 1978 & 5804 & 11868 & $9.07 \mathrm{e}-05$ & $9.07 \mathrm{e}-05$ & $1.232-02$ & $1.23 \mathrm{e}-02$ & 125 \\
\hline 3 & 1190 & 3549 & 11900 & $5.13 \mathrm{e}-06$ & $5.13 \mathrm{e}-06$ & $6.32 \mathrm{e}-04$ & $6.32 \mathrm{e}-04$ & 100 \\
\hline 4 & 804 & 2399 & 12060 & $2.32 \mathrm{e}-07$ & $2.32 \mathrm{e}-07$ & $3.03 \mathrm{e}-05$ & $3.03 \mathrm{e}-05$ & 89 \\
\hline 5 & 572 & 1709 & 12012 & $1.94 \mathrm{e}-08$ & $1.90 \mathrm{e}-08$ & $2.09 \mathrm{e}-06$ & $2.09 \mathrm{e}-06$ & 77 \\
\hline 6 & 426 & 1278 & 11928 & $4.24 \mathrm{e}-09$ & $8.20 \mathrm{e}-10$ & $9.77 \mathrm{e}-08$ & $9.73 \mathrm{e}-08$ & 66 \\
\hline 7 & 330 & 989 & 11880 & $4.16 \mathrm{e}-09$ & $7.79 \mathrm{e}-11$ & $1.32 \mathrm{e}-08$ & 8.31e-09 & 59 \\
\hline 8 & 274 & 820 & 12330 & $4.16 \mathrm{e}-09$ & $3.33 \mathrm{e}-12$ & $1.05 \mathrm{e}-08$ & $3.69 \mathrm{e}-10$ & 54 \\
\hline \multicolumn{9}{|c|}{ DG discretizations on standard meshes } \\
\hline 1 & 4082 & 6222 & 12246 & $3.44 \mathrm{e}-03$ & $3.44 \mathrm{e}-03$ & $2.49 \mathrm{e}-01$ & $2.49 \mathrm{e}-01$ & 176 \\
\hline 2 & 2060 & 3160 & 12360 & $1.08 \mathrm{e}-04$ & $1.08 \mathrm{e}-04$ & $1.34 \mathrm{e}-02$ & $1.34 \mathrm{e}-02$ & 143 \\
\hline 3 & 1156 & 1786 & 11560 & $9.24 \mathrm{e}-06$ & $7.31 \mathrm{e}-06$ & $7.49 \mathrm{e}-04$ & $7.49 \mathrm{e}-04$ & 125 \\
\hline 4 & 816 & 1268 & 12240 & $1.11 \mathrm{e}-05$ & $3.58 \mathrm{e}-07$ & $1.05 \mathrm{e}-04$ & $3.97 \mathrm{e}-05$ & 114 \\
\hline 5 & 596 & 932 & 12516 & $1.61 \mathrm{e}-05$ & $2.22 \mathrm{e}-08$ & $1.50 \mathrm{e}-04$ & $2.29 \mathrm{e}-06$ & 104 \\
\hline 6 & 412 & 648 & 11536 & $3.92 \mathrm{e}-05$ & $1.83 \mathrm{e}-09$ & $3.36 \mathrm{e}-04$ & $1.76 \mathrm{e}-07$ & 90 \\
\hline 7 & 318 & 504 & 11448 & $6.72 \mathrm{e}-05$ & $1.33 \mathrm{e}-10$ & $5.48 \mathrm{e}-04$ & $1.28 \mathrm{e}-08$ & 87 \\
\hline 8 & 280 & 446 & 12600 & $6.67 \mathrm{e}-05$ & $5.25 \mathrm{e}-12$ & $5.58 \mathrm{e}-04$ & $5.32 \mathrm{e}-10$ & 87 \\
\hline \multicolumn{9}{|c|}{ HHO discretizations on agglomerated grids $\left(\mathcal{T}_{h}=\mathcal{T}_{H}, \mathcal{F}_{h}=\mathcal{F}_{h}^{H}\right)$} \\
\hline 0 & 4080 & 19160 & 19160 & $1.83 \mathrm{e}-03$ & $1.83 \mathrm{e}-03$ & $2.31 \mathrm{e}-01$ & $2.31 \mathrm{e}-01$ & 175 \\
\hline 1 & 1978 & 13636 & 27270 & $4.29 \mathrm{e}-04$ & $4.29 \mathrm{e}-04$ & $1.48 \mathrm{e}-02$ & $1.48 \mathrm{e}-02$ & 129 \\
\hline 2 & 1190 & 10673 & 32019 & $7.09 \mathrm{e}-06$ & $7.09 \mathrm{e}-06$ & $9.14 \mathrm{e}-04$ & $9.14 \mathrm{e}-04$ & 100 \\
\hline 3 & 804 & 8846 & 35384 & $7.71 \mathrm{e}-07$ & $7.69 \mathrm{e}-07$ & $5.06 \mathrm{e}-05$ & $5.06 \mathrm{e}-05$ & 84 \\
\hline 4 & 572 & 7575 & 37875 & $2.44 \mathrm{e}-08$ & $2.33 \mathrm{e}-08$ & $3.63 \mathrm{e}-06$ & $3.63 \mathrm{e}-06$ & 71 \\
\hline 5 & 426 & 6596 & 39576 & $3.37 \mathrm{e}-09$ & $2.02 \mathrm{e}-09$ & $1.89 \mathrm{e}-07$ & $1.89 \mathrm{e}-07$ & 63 \\
\hline 6 & 330 & 5915 & 41405 & $4.14 \mathrm{e}-09$ & $9.36 \mathrm{e}-11$ & $1.91 \mathrm{e}-08$ & $1.62 \mathrm{e}-08$ & 57 \\
\hline 7 & 274 & 5412 & 43296 & $4.16 \mathrm{e}-09$ & $5.34 \mathrm{e}-12$ & $1.03 \mathrm{e}-08$ & $7.86 \mathrm{e}-10$ & 52 \\
\hline \multicolumn{9}{|c|}{ HHO discretizations on standard grids } \\
\hline 0 & 4082 & 6222 & 6222 & $1.01 \mathrm{e}-02$ & $1.01 \mathrm{e}-02$ & $2.91 \mathrm{e}-01$ & $2.91 \mathrm{e}-01$ & 145 \\
\hline 1 & 2060 & 3160 & 6320 & $1.53 \mathrm{e}-03$ & $1.53 \mathrm{e}-03$ & $3.92 \mathrm{e}-02$ & $3.92 \mathrm{e}-02$ & 127 \\
\hline 2 & 1156 & 1786 & 5358 & $6.23 \mathrm{e}-05$ & $6.28 \mathrm{e}-05$ & 4.71e-03 & 4.71e-03 & 98 \\
\hline 3 & 816 & 1268 & 5072 & $1.66 \mathrm{e}-05$ & $7.25 \mathrm{e}-06$ & $3.87 \mathrm{e}-04$ & $3.82 \mathrm{e}-04$ & 84 \\
\hline 4 & 596 & 932 & 4660 & $1.62 \mathrm{e}-05$ & $3.83 \mathrm{e}-07$ & $9.92 \mathrm{e}-05$ & $3.18 \mathrm{e}-05$ & 85 \\
\hline 5 & 412 & 648 & 3888 & $3.91 \mathrm{e}-05$ & $3.61 \mathrm{e}-08$ & $2.66 \mathrm{e}-04$ & $3.35 \mathrm{e}-06$ & 90 \\
\hline 6 & 318 & 504 & 3528 & $6.70 \mathrm{e}-05$ & $2.98 \mathrm{e}-09$ & $4.58 \mathrm{e}-04$ & $2.88 \mathrm{e}-07$ & 70 \\
\hline 7 & 280 & 446 & 3568 & $6.67 \mathrm{e}-05$ & $1.81 \mathrm{e}-10$ & $5.38 \mathrm{e}-04$ & $1.62 \mathrm{e}-08$ & 66 \\
\hline
\end{tabular}



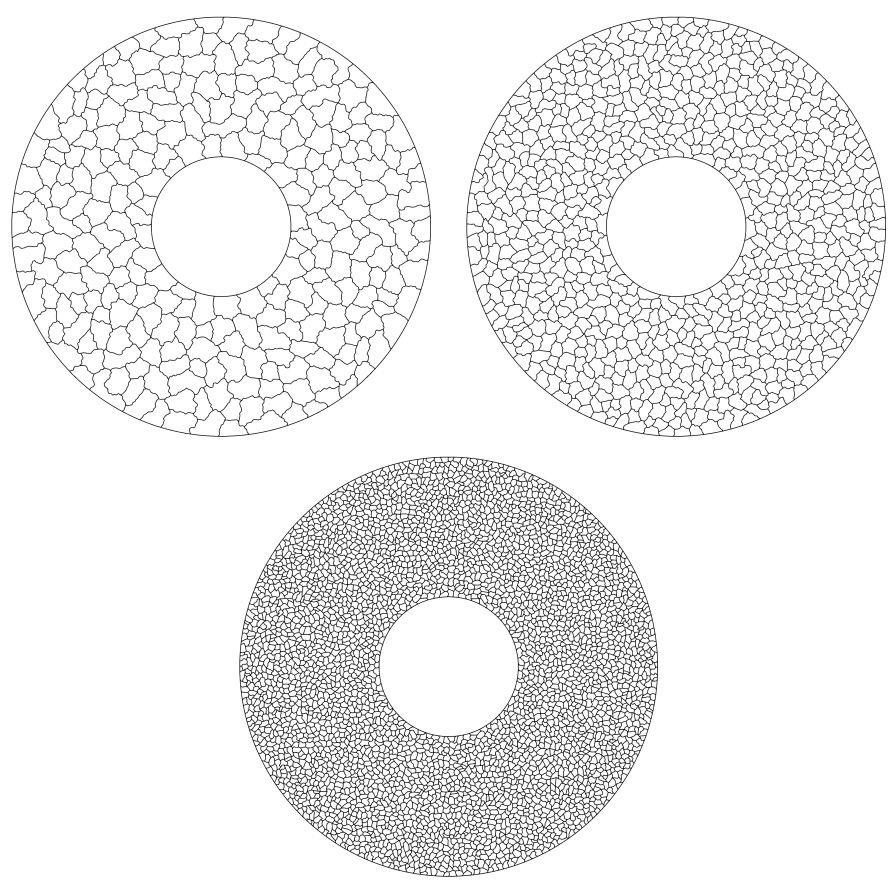

(a) Agglomerated mesh sequence built on top of a 40k six-node triangular mesh.
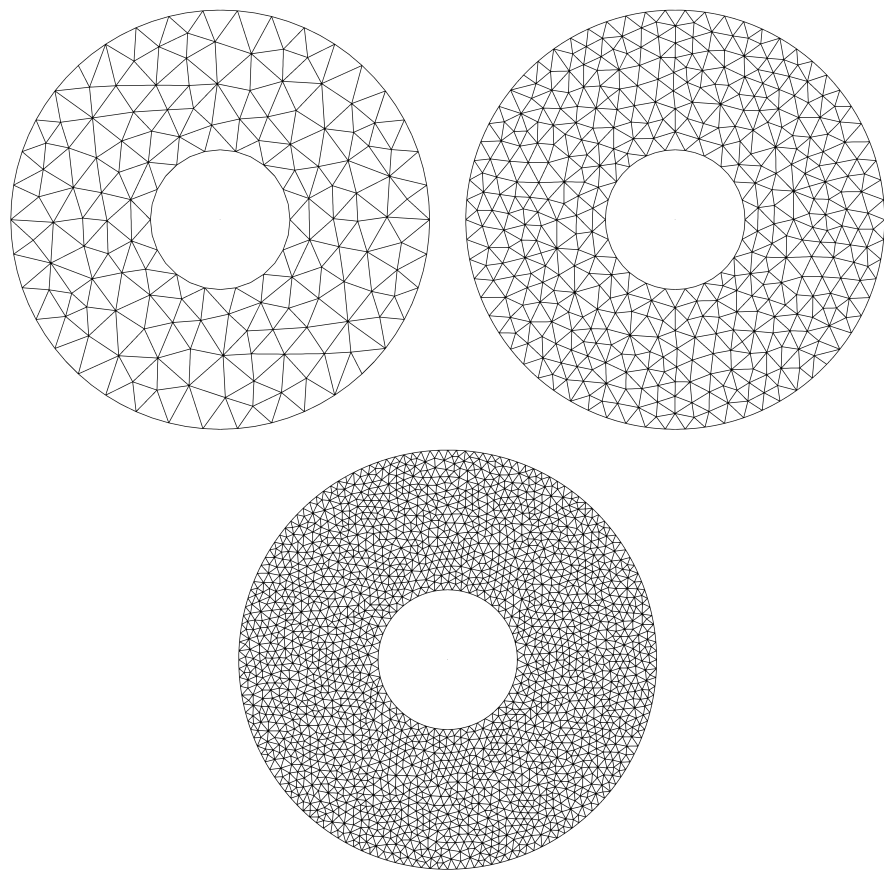

(b) Six-node triangular mesh sequence.

Figure 17: Sequences of meshes of the unit annulus. 

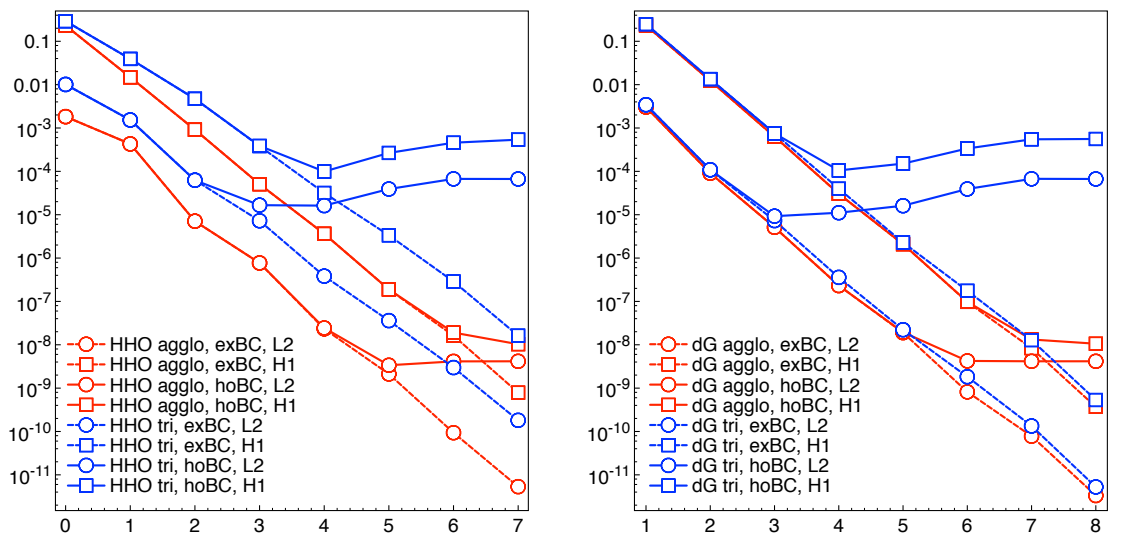

Figure 18: Error versus polynomial degree. $p$-convergence of HHO and DG discretizations on $h$-coarsened agglomerated and regular mesh sequence of Figures $17 \mathrm{a} 17 \mathrm{~b} L^{2}$ and $H^{1}$ measure the error in $L^{2}\left(\Omega_{h}\right)$ norm with respect to the exact solution and the exact solution gradient, respectively, using the boundary condition in $30 \mathrm{a}$ and $30 \mathrm{~b}$ (exBC and hoBC, respectively).

The comparison of $p$-refined HHO and DG discretizations based on the sequences of spaces $\left\{\underline{\mathrm{U}}_{h}^{k, k}\right\}_{k \geqslant 0}$ and $\left\{\mathrm{U}_{h}^{k}\right\}_{k \geqslant 1}$, respectively, shows that HHO methods have a clear advantage in terms of accuracy per DOF in two space dimensions, both on triangular and quadrilateral pathological mesh sequences. In three space dimensions, this advantage is maintained on tetrahedral mesh sequences, while the performance of the two nonconforming methods is comparable on hexahedral mesh sequences. It is interesting to remark that, among standard three dimensional elements shapes (also including e.g. prismatic and pyramidal elements), tetrahedral and hexehedral meshes have the highest and the lowest mesh elements to mesh faces cardinality ratio, respectively. Since the DOF count is face-based for HHO methods and element-based for DG methods, it is no surprise that HHO shines on tetrahedral meshes.

To conclude, asymptotically small discretization errors have been achieved employing high-order HHO and DG discretizations over a unit annulus computational domain with curved boundaries. Remarkably, error reduction is pursued considering high-polynomial degrees over meshes obtained by agglomeration coarsening of a second-order six-node triangular grid obtained with standard mesh generators. Agglomeration-coarsening has been designed to provide a uniform number of DOFs while increasing the polynomial degree in case of $\mathrm{dG}$ discretizations. In view of the discussion in the previous paragraph, DG seems the choice of preference in this context, since on agglomerated mesh sequences the average number of faces per mesh element is typically much larger than on standard grids. Variations of the HHO method that improve its behaviour on agglomerated meshes are currently under study, and will make the object of a future work.

\section{References}

[1] F. Bassi, L. Botti, A. Colombo, S. Rebay, Agglomeration based discontinuous Galerkin discretization of the Euler and Navier-Stokes equations, Computers \& Fluids 61 (2012) 77-85.

[2] X. Zhang, S. Tan, A simple and accurate discontinuous Galerkin scheme for modeling scalar-wave 
propagation in media with curved interfaces, Geophysics 80 (2) (2015) T83-T89. doi:10.1190/ geo2014-0164.1

[3] X. Zhang, A curved boundary treatment for discontinuous Galerkin schemes solving time dependent problems, J. Comput. Phys. 308 (2016) 153 - 170. doi:10.1016/j.jcp.2015.12.036.

[4] G. J. Gassner, F. Lörcher, C.-D. Munz, J. S. Hesthaven, Polymorphic nodal elements and their application in discontinuous galerkin methods, J. Comput. Phys. 228 (2009) 1573-1590.

[5] F. Bassi, L. Botti, A. Colombo, D. A. Di Pietro, P. Tesini, On the flexibility of agglomeration based physical space discontinuous Galerkin discretizations, J. Comput. Phys. 231 (1) (2012) 4565. doi:10.1016/j.jcp.2011.08.018.

6] P. F. Antonietti, S. Giani, P. Houston, $h p$-version composite discontinuous Galerkin methods for elliptic problems on complicated domains, SIAM J. Sci. Comput. 35 (3) (2013) A1417-A1439. doi:10.1137/120877246.

[7] P. F. Antonietti, A. Cangiani, J. Collis, Z. Dong, E. H. Georgoulis, S. Giani, P. Houston, Review of Discontinuous Galerkin Finite Element Methods for Partial Differential Equations on Complicated Domains, Springer International Publishing, Cham, 2016, pp. 281-310. doi: 10.1007/978-3-319-41640-3_9

[8] D. A. Di Pietro, A. Ern, A hybrid high-order locking-free method for linear elasticity on general meshes, Comput. Meth. Appl. Mech. Engrg. 283 (2015) 1-21. doi:10.1016/j.cma.2014.09.009

[9] D. A. Di Pietro, A. Ern, S. Lemaire, An arbitrary-order and compact-stencil discretization of diffusion on general meshes based on local reconstruction operators, Comput. Meth. Appl. Math. 14 (4) (2014) 461-472, open access (editor's choice). doi:10.1515/cmam-2014-0018

[10] L. Beirão da Veiga, F. Brezzi, A. Cangiani, G. Manzini, L. D. Marini, A. Russo, Basic principles of virtual element methods, Math. Models Methods Appl. Sci. 23 (01) (2013) 199-214. doi:10.1142/ S0218202512500492.

[11] T. Warburton, A low-storage curvilinear discontinuous Galerkin method for wave problems, SIAM J. Sci. Comput. 35 (4) (2013) A1987-A2012. doi:10.1137/120899662

[12] J. Chan, R. J. Hewett, T. Warburton, Weight-adjusted discontinuous Galerkin methods: curvilinear meshes, SIAM J. Sci. Comput. 39 (6) (2017) A2395-A2421. doi:10.1137/16M1089198

[13] J. Chan, R. J. Hewett, T. Warburton, Weight-adjusted discontinuous Galerkin methods: wave propagation in heterogeneous media, SIAM J. Sci. Comput. 39 (6) (2017) A2935-A2961. doi: 10.1137/16M1089186

[14] C. Nguyen, J. Peraire, An adaptive shock-capturing HDG method for compressible flows, in: 20th AIAA Computational Fluid Dynamics Conference, Honolulu, Hawaii, American Institute of Aeronautics and Astronautics, 2011. doi:10.2514/6.2011-3060

[15] A. Jaust, J. Schütz, M. Woopen, An HDG Method for Unsteady Compressible Flows, Springer International Publishing, Cham, 2015, pp. 267-274. doi:10.1007/978-3-319-19800-2_23

[16] C. Gürkan, E. Sala-Lardies, M. Kronbichler, S. Fernández-Méndez, extended hybridizable discontinous galerkin (x-hdg) for void problems, J. Sci. Comput. 66 (3) (2016) 1313-1333. doi: 10.1007/s10915-015-0066-8

[17] K. J. Fidkowski, A hybridized discontinuous galerkin method on mapped deforming domains, Computers \& Fluids 139 (2016) 80 - 91, 13th USNCCM International Symposium of High-Order Methods for Computational Fluid Dynamics - A special issue dedicated to the 60th birthday of Professor David Kopriva. doi:10.1016/j.compfluid.2016.04.004

[18] C. Michoski, J. Chan, L. Engvall, J. Evans, Foundations of the blended isogeometric discontinuous galerkin (bidg) method, Comp. Methods Appl. Mech. Engrg. 305 (2016) 658 - 681. doi:10.1016/ j.cma.2016.02.015.

[19] L. Beirão da Veiga, A. Russo, G. Vacca, The Virtual Element Method with curved edges, submitted (November 2017). URL https://arxiv.org/abs/1711.04306

[20] S. C. Brenner, L. R. Scott, The Mathematical Theory of Finite Element Methods, 3rd Edition, Springer-Verlag, New York-Berlin-Heidelberg, 2008

[21] G. E. Karniadakis, S. Sherwin, Spectral $/ h p$ Element Methods for Computational Fluid Dynamics, Numerical Mathematics and Scientific Computation, Oxford University Press, USA, 2005.

[22] F. Bassi, A. Crivellini, D. A. Di Pietro, S. Rebay, An artificial compressibility flux for the discontinuous Galerkin solution of the incompressible Navier-Stokes equations, J. Comput. Phys. 218 (2006) 794-815. doi:10.1016/j.jcp.2006.03.006

[23] D. A. Di Pietro, A. Ern, Mathematical Aspects of Discontinuous Galerkin Methods, Vol. 69 of Maths \& Applications, Springer-Verlag, 2011. doi:10.1007/978-3-642-22980-0

[24] D. A. Di Pietro, J. Droniou, A Hybrid High-Order method for Leray-Lions elliptic equations on 
general meshes, Math. Comp. 86 (307) (2017) 2159-2191. doi:10.1090/mcom/3180

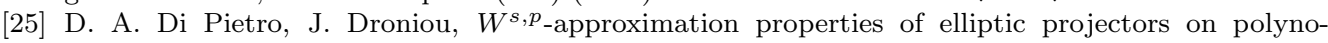
mial spaces, with application to the error analysis of a Hybrid High-Order discretisation of Leray-Lions problems, Math. Models Methods Appl. Sci. 27 (05) (2017) 879-908. doi:10.1142/ S0218202517500191.

[26] T. Dupont, R. Scott, Polynomial approximation of functions in Sobolev spaces, Math. Comp. 34 (150) (1980) 441-463.

[27] L. Botti, Influence of reference-to-physical frame mappings on approximation properties of discontinuous piecewise polynomial spaces, Journal of Scientific Computing 52 (3) (2012) 675-703.

[28] D. N. Arnold, D. Boffi, R. S. Falk, Approximation by quadrilateral finite elements, Math. Comp. 71 (239) (2002) 909-922.

[29] F. Bassi, S. Rebay, G. Mariotti, S. Pedinotti, M. Savini, A high-order accurate discontinuous finite element method for inviscid and viscous turbomachinery flows, in: R. Decuypere, G. Dibelius (Eds.), Proceedings of the 2nd European Conference on Turbomachinery Fluid Dynamics and Thermodynamics, Technologisch Instituut, Antwerpen, Belgium, 1997, pp. 99-108.

[30] F. Brezzi, G. Manzini, D. Marini, P. Pietra, A. Russo, Discontinuous Galerkin approximations for elliptic problems, Numerical Methods for Partial Differential Equations 16 (2000) 365-378.

[31] D. A. Di Pietro, R. Tittarelli, Lectures from the fall 2016 thematic quarter at Institut Henri Poincaré Springer, 2017, Ch. An introduction to Hybrid High-Order methods, to appear. URL http://arxiv . org/abs/1703.05136

[32] D. A. Di Pietro, A. Ern, S. Lemaire, An arbitrary-order and compact-stencil discretization of diffusion on general meshes based on local reconstruction operators, Computational Methods in Applied Mathematics 14 (4) (2017) 461 - 472. doi:10.1515/cmam-2014-0018

[33] M. Cicuttin, D. A. Di Pietro, A. Ern, Implementation of Discontinuous Skeletal methods on arbitrary-dimensional, polytopal meshes using generic programming, published online (2017). doi:10.1016/j.cam.2017.09.017

[34] F. Bassi, L. Botti, A. Colombo, Agglomeration based physical frame dG discretizations: An attempt to be mesh free, Math. Models Methods Appl. Sci. 24 (08) (2014) 1495-1539. doi: 10.1142/S0218202514400028

[35] J. Droniou, R. Eymard, T. Gallouët, R. Herbin, A unified approach to mimetic finite difference, hybrid finite volume and mixed finite volume methods, Math. Models Methods Appl. Sci. 20 (2) (2010) $1-31$.

[36] F. Brezzi, K. Lipnikov, M. Shashkov, Convergence of mimetic finite difference method for diffusion problems on polyhedral meshes with curved faces, Math. Models Methods Appl. Sci. 16 (2) (2006) 275-297.

[37] K. Gobbert, S. Yang, Numerical Demonstration of Finite Element Convergence for Lagrange Elements in COMSOL Multiphysics, in: Proceedings of the COMSOL Conference 2008 Boston, 2008.

[38] T. Toulorge, C. Geuzaine, J.-F. Remacle, J. Lambrechts, Robust untangling of curvilinear meshes, J. Comput. Phys. 254 (2013) 8 - 26. doi:10.1016/j.jcp.2013.07.022 